\title{
Genus 4 trigonal reduction of the Benney equations
}

\author{
Sadie Baldwin and John Gibbons \\ Imperial College \\ 180 Queen's Gate \\ London SW7 2BZ \\ E-mail: sadie.baldwin@imperial.ac.uk, j.gibbons@imperial.ac.uk
}

\begin{abstract}
It was shown by Gibbons and Tsarev (1996 Phys. Lett. A 211 19, 1999 Phys. Lett. A 258 263) that $N$-parameter reductions of the Benney equations correspond to particular $N$-parameter families of conformal maps.

In recent papers (J. Phys. A: Math. Gen. 36 No 31 (8 August 2003) 8393-8417), (J. Phys. A: Math. Gen. 37 No 20 (21 May 2004) 5341-5354), the present authors have constructed examples of such reductions where the mappings take the upper half $p$-plane to a polygonal slit domain in the $\lambda$-plane. In those cases the mapping function was expressed in terms of the derivatives of Kleinian $\sigma$ functions of hyperelliptic curves, restricted to the 1-dimensional stratum $\Theta_{1}$ of the $\Theta$-divisor. This was done using an extension of the method given in Enolskii et al (2003 J. Nonlinear Sci. 13 157) extended to a genus 3 curve (V Z Enolski and J Gibbons, Addition theorems on the strata of the theta divisor of genus three hyperelliptic curves, (in preparation)). Here, we use similar ideas, but now applied to a trigonal curve of genus 4 . Fundamental to this approach is a family of differential relations which $\sigma$ satisfies on the divisor. Again, it is shown that the mapping function is expressible in terms of quotients of derivatives of $\sigma$ on the divisor $\Theta_{1}$. One significant by-product is an expansion of the leading terms of the Taylor series of $\sigma$ for the given family of $(3,5)$ curves; to the best of the authors' knowledge, this is new.
\end{abstract}

PACS numbers: 02.30.Ik, 02.30.Jr, 02.30.Zz

\section{Introduction}

\subsection{Reductions of the Benney Moment Equations}

The Benney equations [1] are an example of a system of hydrodynamic type with infinitely many degrees of freedom. These can be written as a Vlasov equation [2], $[3]$

$$
\frac{\partial f}{\partial t_{2}}+p \frac{\partial f}{\partial x}-\frac{\partial A_{0}}{\partial x} \frac{\partial f}{\partial p}=0
$$

Here $f=f(x, p, t)$ is a distribution function. The moments $A_{n}$ are defined by

$$
A_{n}=\int_{-\infty}^{\infty} p^{n} f \mathrm{~d} p
$$


Following [4], we let $\lambda_{\mathrm{R}}(x, p, t)$, be given by the integral

$$
\lambda_{\mathrm{R}}=p+\mathrm{P} \int_{-\infty}^{\infty} \frac{f\left(x, p^{\prime}, t\right)}{\left(p-p^{\prime}\right)} \mathrm{d} p^{\prime}
$$

where $\mathrm{P}$ denotes the principal value. Comparing the first derivatives of $\lambda_{\mathrm{R}}(x, p, t)$, we obtain the PDE

$$
\frac{\partial \lambda_{\mathrm{R}}}{\partial t}+p \frac{\partial \lambda_{\mathrm{R}}}{\partial x}=\frac{\partial \lambda_{\mathrm{R}}}{\partial p}\left(\frac{\partial p}{\partial t}+p \frac{\partial p}{\partial x}+\frac{\partial A_{0}}{\partial x}\right) .
$$

If we hold $\lambda_{\mathrm{R}}$ constant in (4), then this gives the conservation equation

$$
\frac{\partial p}{\partial t}+\frac{\partial}{\partial x}\left(\frac{1}{2} p^{2}+A_{0}\right)=0
$$

Alternatively, if we now hold $p$ constant in (4), we obtain

$$
\frac{\partial \lambda_{\mathrm{R}}}{\partial t}+p \frac{\partial \lambda_{\mathrm{R}}}{\partial x}-\frac{\partial A_{0}}{\partial x} \frac{\partial \lambda_{\mathrm{R}}}{\partial p}=0
$$

which is a Vlasov equation of the same form as (1). Thus (1) and (6) have the same characteristics. Any function of $\lambda_{\mathrm{R}}$ and $f$ must satisfy the same equation.

Suppose further that for some point $p=\hat{p}_{i}(x, t), \quad \lambda_{\mathrm{R}}\left(\hat{p}_{i}\right)=\hat{\lambda}_{i}(x, t)$ we have:

$$
\left.\frac{\partial \lambda_{\mathrm{R}}}{\partial p}\right|_{p=\hat{p}_{i}}=0
$$

then substituting

$$
\left.\frac{\partial \lambda_{\mathrm{R}}}{\partial t}\right|_{p=\hat{p}_{i}}=\frac{\partial \hat{\lambda}_{i}}{\partial t} \quad \text { and }\left.\quad \frac{\partial \lambda_{\mathrm{R}}}{\partial x}\right|_{p=\hat{p}_{i}}=\frac{\partial \hat{\lambda}_{i}}{\partial x}
$$

into (4) gives

$$
\frac{\partial \hat{\lambda}_{i}}{\partial t}+\hat{p}_{i} \frac{\partial \hat{\lambda}_{i}}{\partial x}=0
$$

We say that $\hat{\lambda}_{i}$ is a Riemann invariant with characteristic speed $\hat{p}_{i}$.

We are interested in the case where the function $\lambda_{\mathrm{R}}(p, x, t)$ is such that only $N$ of the moments $A_{n}$ are independent. Then it was shown in [5] that there are $N$ characteristic speeds, assumed real and distinct, and $N$ corresponding Riemann invariants $\left(\hat{p}_{i}, \hat{\lambda}_{i}\right)$. Then Benney's equations reduce to a diagonal system of hydrodynamic type with finitely many, $N$, dependent variables $\hat{\lambda}_{i}$, satisfying:

$$
\frac{\partial \hat{\lambda}_{i}}{\partial t}+\hat{p}_{i}(\hat{\lambda}) \frac{\partial \hat{\lambda}_{i}}{\partial x}=0 \quad(i=1,2, \ldots, N) .
$$

Such a system is called a reduction of Benney's equations.

The construction of a general family of solutions for equations of this type was outlined in [5] and [6]. Instead of considering the principal value integral (3), we now define a new function $\lambda_{+}(x, p, t)$ :

$$
\lambda_{+}(x, p, t)=p+\int_{\Upsilon} \frac{f\left(x, p^{\prime}, t\right)}{p-p^{\prime}} \mathrm{d} p^{\prime}
$$


where $\Upsilon$ is an indented contour passing below the point $p$. This function has the same asymptotics as $\lambda_{\mathrm{R}}(x, p, t)$, provided all the moments $A_{n}$ exist, and it can be analytically continued throughout the upper half $p$-plane, provided that $f$ is Hölder continuous.

We now suppose that the relation $f=F\left(\lambda_{R}\right)$ holds in some region of the $(x, p)$ plane at some time $t$, and that $f=0$ outside this region. Then since both (1) and (6) have the same characteristics, the relation will be preserved by the dynamics. In this case the definition for $\lambda_{+}(8)$ becomes a nonlinear singular integral equation:

$$
\lambda_{+}(x, p, t)=p+\int_{\Upsilon} \frac{F\left(\lambda_{R}\left(x, p^{\prime}, t\right)\right)}{p-p^{\prime}} \mathrm{d} p^{\prime} .
$$

Some solutions to (9) can be described in terms of a conformal mapping of a slit domain. We take the upper half $\lambda$-plane, $\Gamma_{+}$, and draw a Jordan $\operatorname{arc} c$ in $\Gamma_{+}$starting from a point, $\lambda_{1}^{0}$, on the real axis. We then fix an arbitrary point on this arc, $\hat{\lambda}_{1}$, and make a slit $\gamma_{1}$ running along the arc from $\lambda_{1}^{0}$ to $\hat{\lambda}_{1}$.

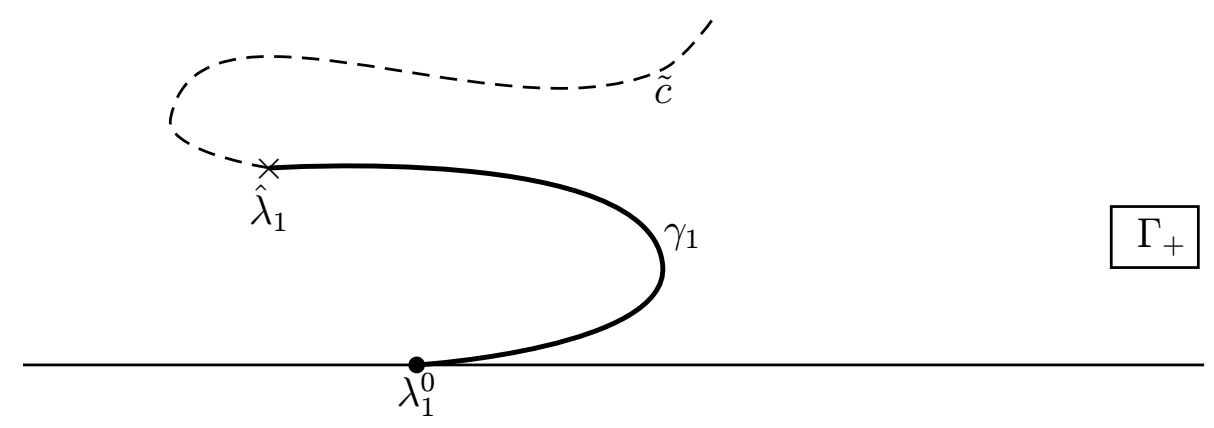

Figure 1. The slit $\gamma_{1}$ on the Jordan $\operatorname{arc} c=\gamma_{1} \cup \tilde{c}$.

Note that the slit $\gamma_{1}$ in figure 1 is given by the relation

$$
\operatorname{Im}\left(\lambda_{+}\right)=-\pi F\left(\operatorname{Re}\left(\lambda_{+}\right)\right)
$$

and so, for consistency, $F$ must be continuous with $F \leq 0$. The function $p\left(\lambda_{+}, \hat{\lambda}_{1}\right)$ is then determined uniquely by the following properties.

(i) $p\left(\lambda_{+}, \hat{\lambda}_{1}\right)$ has a branch point at $\hat{\lambda}_{1}$, that is

$$
p \sim \hat{p}+c\left(\lambda-\hat{\lambda}_{1}\right)^{\frac{1}{2}}+\mathrm{O}\left(\lambda-\hat{\lambda}_{1}\right) .
$$

(ii) $p\left(\lambda_{+}, \hat{\lambda}_{1}\right)$ is real on the real $\lambda_{+}$-axis and on both sides of $\gamma_{1}$.

(iii) $p\left(\lambda_{+}, \hat{\lambda}_{1}\right)$ is analytic in the cut half plane $\Gamma_{+}$.

(iv) As $|\lambda| \rightarrow \infty$, with $\operatorname{Im}\left(\lambda_{+}\right) \geq 0, p\left(\lambda_{+}, \hat{\lambda}_{1}\right)$ has the expansion

$$
p\left(\lambda_{+}, \hat{\lambda}_{1}\right) \sim \lambda_{+}+\mathrm{O}\left(\frac{1}{\lambda_{+}}\right) .
$$

The evolution of $p$ is then given by (5); expanding near $\hat{\lambda}_{1}$ gives:

$$
\frac{\partial \hat{\lambda}_{1}}{\partial t}+\hat{p} \frac{\partial \hat{\lambda}_{1}}{\partial x}=0
$$


Thus $\hat{\lambda}_{1}$ is a Riemann invariant with characteristic speed $\hat{p}=p\left(\hat{\lambda}_{1}\right)$.

It is possible to generalize this construction to $N$ non-intersecting slits. Here, each of the slits $\gamma_{i}$ is made along a fixed path starting on the real $\lambda_{+}$-axis and ending in a branch point $\hat{\lambda}_{i}$. Again, $\hat{\lambda}_{i}$ are the Riemann invariants of the system with associated characteristic speeds $p\left(\hat{\lambda}_{i}\right)$ and the slits $\gamma_{i}$ are given by

$$
\operatorname{Im}\left(\lambda_{+}\right)=-\pi F_{i}\left(\operatorname{Re}\left(\lambda_{+}\right)\right)
$$

where $F_{i} \leq 0$ are continuous functions.

In the particular case that the slits are all straight line segments, making angles with the real $\lambda$ axis which are rational multiples of $\pi$, the usual Schwartz-Christoffel construction gives a mapping function of the form:

$$
\lambda_{+}=p+\int_{-\infty}^{p}\left[\phi\left(p^{\prime}\right)-1\right] \mathrm{dp}^{\prime}
$$

where $\phi\left(p^{\prime}\right)$ is some algebraic function. In this case it is natural to consider this expression as an integral of a second-kind differential on the corresponding algebraic curve. This approach was used in [7], [8] and [9], where the slits were all at right angles to the real axis, and the corresponding curves were then elliptic or hyperelliptic. The question thus arises whether a similar approach is equally useful for a curve which is not of this type; here we look at a particular example, where the underlying family of curves are trigonal.

While the resulting formula (103) is clearly highly transcendental, it is remarkable that all known examples of such explicit representations of Schwarz-Christoffel slit mappings may be written as rational functions of derivatives of $\sigma$-functions for the corresponding algebraic curve. The principal advantage of such an approach is that the original Schwartz-Christoffel integral depends on many parameters, which must satisfy integral constraints. In the present trigonal case for example, it depends on 10 parameters, satisfying 6 constraints - it is thus very hard to use the integral representation to calculate the properties of the reduced system, without evaluating the integral anyway. Calculating the Hamiltonian structure, for example, in terms of the $\sigma$ function representation may well be more tractable; work on this is continuing. Some recent closely related work on expressing the analogous hyperelliptic mappings in terms of automorphic functions, by Crowdy [10], [11] suggests further generalisations may be possible. In that representation, the constraints are satisfied automatically, and the mapping no longer contains spurious parameters, depending only upon the dynamical variables.

In a series of papers by Wiegmann, Krichever, Mineev-Weinstein, Zabrodin and co-workers, (see, e.g. [12],[13]), a related problem, in a sense inverse to this one, is addressed. There, families of conformal maps are constructed in terms of the solutions of dispersionless integrable hierarchies, and these are further related to the solutions of random matrix models. The detailed connections between that work and this still remain to be clarified. It is clear, however, that the topics of conformal mappings and of dispersionless integrable hierarchies are intimately connected. 


\section{A Trigonal Reduction}

To motivate the calculations which follow, we consider reductions where the slits are straight line segments making angles of $\pi / 3$ or $2 \pi / 3$ with the real axis, leading to a trigonal curve. There is one elementary example with this slit geometry, leading to the dispersionless Boussinesq hierarchy [14]. Here the mapping is

$$
\lambda_{+}=\left(p^{3}+3 A_{0} p+3 A_{1}\right)^{1 / 3}=\left(\left(p-P_{1}\right)\left(p-P_{2}\right)\left(p-P_{3}\right)\right)^{1 / 3},
$$

and the two slits have fixed base point at the origin, which is the image of the three points $\left\{P_{1}, P_{2}, P_{3}\right\}$. See figures (2) and (3). We should point out that although the mapping itself is written in elementary functions, the curve is non-trivial, having genus 1.

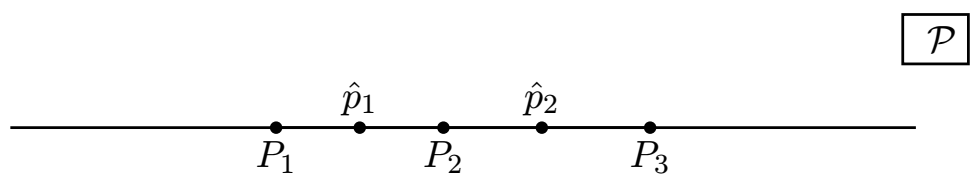

Figure 2. The $p$-plane.

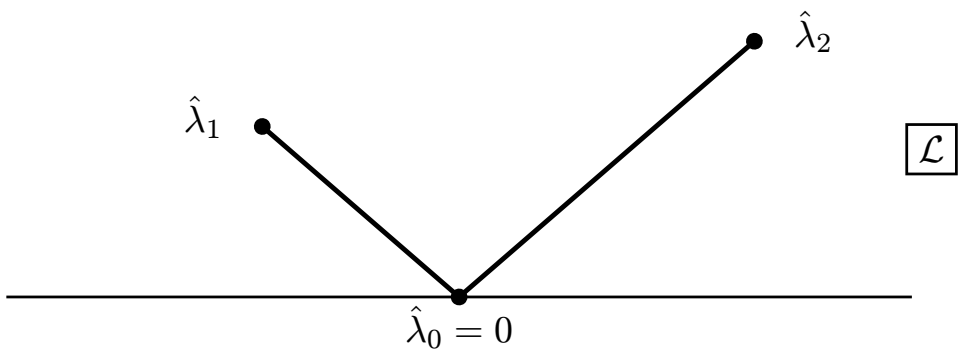

Figure 3. The $\lambda$-plane associated with figure 2 .

Let us now consider a similar reduction, but instead with 2 pairs of slits, as shown in Figure(4) where the conformal mapping $\lambda: \mathcal{P} \rightarrow \mathcal{L}$ is constructed as follows. We define $\mathcal{P}$ to be the upper half $p$-plane with 10 points marked on the real axis. These satisfy

$$
P_{1}<\hat{p}_{1}<P_{2}<\hat{p}_{2}<P_{3}<P_{4}<\hat{p}_{3}<P_{5}<\hat{p}_{4}<P_{6} .
$$

The domain $\mathcal{L}$ is the upper half $\lambda$-plane with 2 pairs of slits on it, as in figure ( 5$)$. The first pair of slits radiate at 60 degree angles from the fixed real point $\lambda_{0}^{1}$; The end points of these slits move along the radial lines and are labelled $\hat{\lambda}_{1}$ and $\hat{\lambda_{2}}$. A second pair of slits is arranged similarly, radiating at 60 degree angles from the fixed real point $\lambda_{0}^{4}$. Here, the variable end points are labelled $\hat{\lambda}_{3}$ and $\hat{\lambda}_{4}$. As in the hyperelliptic cases, the 
point $\hat{\lambda}_{i}$ is the Riemann invariant associated with the characteristic speed $\hat{p}_{i}$. By setting $\lambda\left(P_{i}\right)=\lambda_{i}$ and imposing the conditions

$$
\begin{aligned}
& \lambda_{1}=\lambda_{2}=\lambda_{3}=\lambda_{0}^{1}, \\
& \lambda_{4}=\lambda_{5}=\lambda_{6}=\lambda_{0}^{4},
\end{aligned}
$$

it follows that $\mathcal{L}$ is a slit domain of the form shown in figure (5).

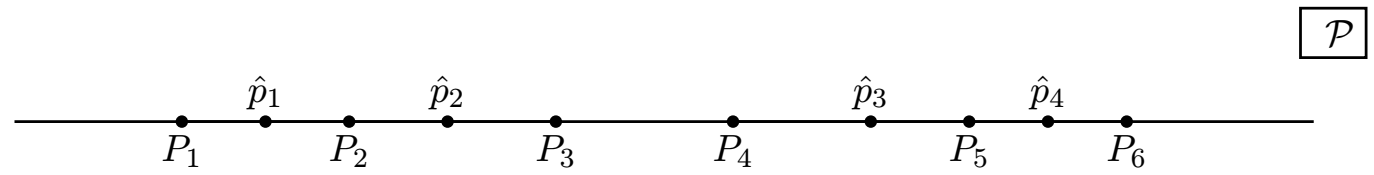

Figure 4. The $p$-plane.

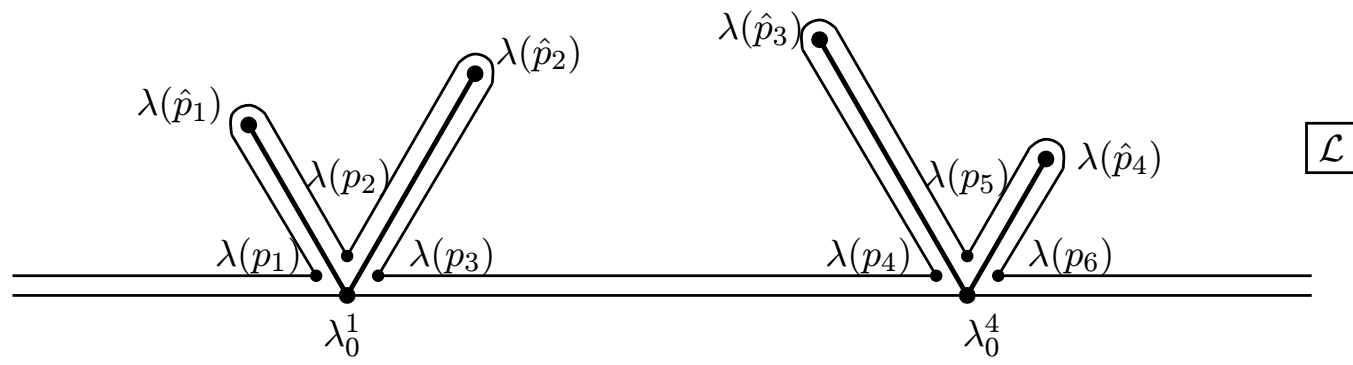

Figure 5. The $\lambda$-plane associated with figure 4 .

The mapping $\lambda: \mathcal{P} \rightarrow \mathcal{L}$ can then be given in the Schwartz-Christoffel form:

$$
\lambda(p)=p+\int_{\infty}^{p}\left(\varphi\left(p^{\prime}\right)-1\right) \mathrm{d} p^{\prime} .
$$

where

$$
\varphi(p)=\frac{\prod_{i=1}^{4}\left(p-\hat{p}_{i}\right)}{\left[\prod_{i=1}^{6}\left(p-P_{i}\right)\right]^{2 / 3}}=\frac{\prod_{i=1}^{4}\left(p-\hat{p}_{i}\right)}{y^{2}},
$$

where

$$
y^{3}=\left(\prod_{i=1}^{6}\left(p-P_{i}\right)\right) .
$$

From the conditions (12), we see that once the base points $\lambda_{0}^{1}$ and $\lambda_{0}^{4}$ have been fixed, the mapping $\lambda(p)$ is a function of 4 independent real parameters. As in the hyperelliptic case, it is natural to take these to be the variable imaginary parts of the slit ends: $\Im\left(\lambda\left(\hat{p}_{i}\right)\right), i=1,2,3,4$.

From the construction of the conformal mappings, we also have the property

$$
\lim _{p \rightarrow \infty} \varphi(p) \sim 1+\mathrm{O}\left(\frac{1}{p^{2}}\right) .
$$


This provides a relation between the characteristic speeds $\hat{p}_{j}$ and the fixed points $P_{i}$. We have

$$
\lim _{p \rightarrow \infty} \varphi(p)=1+\left(\frac{2}{3} \sum_{i=1}^{6} P_{i}-\sum_{i=1}^{4} \hat{p}_{i}\right) \frac{1}{p}+\mathrm{O}\left(\frac{1}{p^{2}}\right)
$$

and so

$$
\sum_{i=1}^{4} \hat{p}_{i}=\frac{2}{3} \sum_{i=1}^{6} P_{i}
$$

Following the process used in the hyperelliptic cases, we now define the Riemann surface $\Gamma$ :

$$
\Gamma=\left\{(p, y) \in \mathbb{C}^{2}: y^{3}=\left(\prod_{i=1}^{6}\left(p-P_{i}\right)\right)\right\}
$$

We will then be able to use the properties of this surface to evaluate the integral $\lambda(p)$.

This is a $(3,6)$-curve and so relates each point $p$, except the branch points $P_{i}$, to three values in the complex plane and so the Riemann surface for (15) consists of three sheets, triply branched at the points $P_{i}$.

For all $p$ in the finite plane, other than the branch points, each branch of the function $y(p)$ is finite and so the curve is regular here, and $p$ is a good local parameter at such points. However, if we evaluate $y$ along a contour encircling the point $P_{i}$, the values at the end points differ by a factor of

$$
\omega=\exp (2 i \pi / 3) \text {. }
$$

Hence, the $P_{i}$ are regular branch points of order 3 . The local co-ordinates at the branch points are

$$
\xi=\left(p-P_{i}\right)^{1 / 3} \quad(i=1, \ldots, 6) .
$$

In the neighbourhood of $P_{i}, y$ is an analytic function of the corresponding local coordinate $\xi$.

We may describe the Riemann surface more precisely, and label the different sheets, by noting that:

$$
\frac{y}{p^{2}} \rightarrow \exp \left(2 \pi i \frac{k-1}{3}\right) \quad \text { as } \quad|p| \rightarrow \infty,
$$

where $k=1,2$ or 3 . The different sheets are joined along the real intervals (the cuts) $\left[P_{1}, P_{2}\right],\left[P_{2}, P_{3}\right],\left[P_{4}, P_{5}\right]$ and $\left[P_{5}, P_{6}\right]$. Specifically, as $p$ passes from a point on the upper side of $\left[P_{2}, P_{3}\right]$ or $\left[P_{5}, P_{6}\right]$ to the lower side, $y$ moves from sheet $k$ to sheet $(k+1) \bmod 3$, and as $p$ passes from a point on the upper side of $\left[P_{1}, P_{2}\right]$ or $\left[P_{4}, P_{5}\right]$ to the lower side, $y$ moves from sheet $k$ to sheet $(k-1) \bmod 3$. The branch cuts, and the connections between the different sheets, are shown in figure (6). The $k$-th sheet is completed by adding a point at infinity, denoted $\infty_{k}$, where a good local co-ordinate is $\xi=1 / p$. Expanding $y(p)$ in terms of this local co-ordinate gives

$$
y(p) \simeq \exp (2 \pi i(k-1) / 3)\left(\frac{1}{\xi^{2}}-\left(\frac{1}{3} \sum_{i=1}^{6} P_{i}\right) \frac{1}{\xi}+\mathrm{O}(1)\right)
$$


and so at each of the 3 points at infinity the function $y$ has poles of order 2 .

Definition 2.1 Any Riemann surface $\mathbf{R}$ given by

$$
y^{n}=Q_{m}(x)
$$

where $n$ is an integer and $Q_{m}$ is a polynomial of order $m$, is called a cyclic $(n, m)$ Riemann surface.

Since all the $n$ sheets have common branch points, at the zeroes of $Q_{m}(x)$, and all branch points are ramified in the same way, these curves are much simpler than more general examples. Thus in our example, the curve $\Gamma$ :

$$
y^{3}=\prod_{i=1}^{6}\left(p-P_{i}\right)
$$

is a cyclic $(3,6)$-curve.

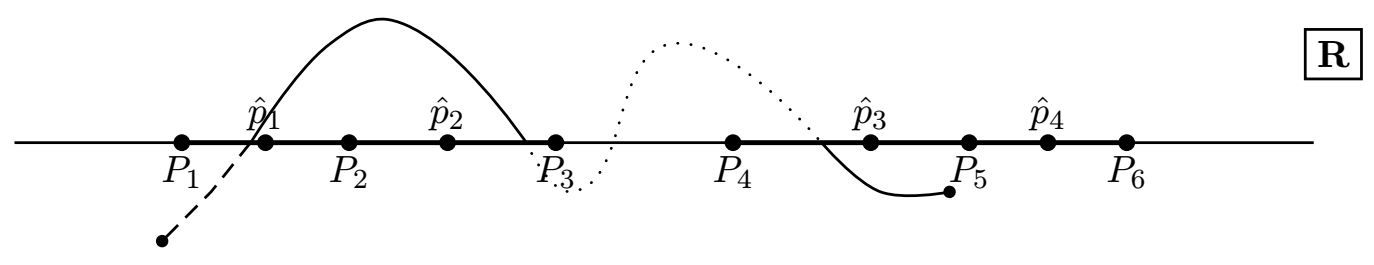

Figure 6. The cyclic trigonal Riemann surface $\Gamma$. The bold lines are cuts on the surface. The solid curve is on sheet 1 , the dashed curve on sheet 2 and the dotted curve on sheet 3 .

\subsection{Properties of the cyclic trigonal Riemann surface}

We now investigate some of the properties of the Riemann surface $\Gamma$. Some key results for trigonal Riemann surfaces have been found by Eilbeck, Enolski and Leykin, [15], by Buchstaber, Enolski and Leykin, [16], which consider $(3,4)$ surfaces in detail, and recently by Ônishi [17], who finds formulae holding on cyclic $(3,4)$ surfaces, while Matsumoto [18] has looked at trigonal curves with 6 branch points, as in our case. Our approach follows the method of [15] and [16] closely.

First it is necessary to calculate the genus of the curve, and to define a basis of $\mathfrak{a}$ and $\mathfrak{b}$ cycles. From the Riemann-Hurwitz theorem, the genus of a cyclic $(n, l)$-curve is given by

$$
2 g=2-2 n+l(n-1)
$$

giving in this case, with $n=3, l=6$,

$$
g=1-3+6.2 / 2=4 .
$$


We can thus define a basis of cycles on $\mathbf{R}$ consisting of four $\mathfrak{a}$ and four $\mathfrak{b}$ cycles. These must have intersection index given by:

$$
\mathfrak{a}_{i} \circ \mathfrak{a}_{j}=0, \quad \mathfrak{a}_{i} \circ \mathfrak{b}_{j}=\delta_{i j}, \quad \mathfrak{b}_{i} \circ \mathfrak{b}_{j}=0,
$$

where $\delta_{i j}$ is the Kronecker delta. A suitable set of cycles for this first homology basis,

$$
\mathrm{H}_{1}(\mathbf{R}, \mathbb{Z})=\left\{\mathfrak{a}_{1}, \mathfrak{a}_{2}, \mathfrak{a}_{3}, \mathfrak{a}_{4} ; \mathfrak{b}_{1}, \mathfrak{b}_{2}, \mathfrak{b}_{3}, \mathfrak{b}_{4}\right\},
$$

is shown in [18].

To identify a basis of holomorphic differentials on $\Gamma$ we need to calculate the Weierstrass gap sequence [19] for the curve. This process is simplified if the orders of $y$ and $p$ are co-prime, that is, if the curve is in canonical form. To achieve this we transform the curve by sending one of the branch points, $P_{6}$, to infinity, using the invertible rational map:

$$
\begin{aligned}
& p=P_{6}-\frac{1}{t}, \\
& P_{i}=P_{6}-\frac{1}{T_{i}} \quad i=1 \ldots 5, \\
& s=y t^{2} K \\
& K^{3}=\prod_{i=1}^{5}\left(P_{6}-P_{i}\right) .
\end{aligned}
$$

For the curve $\Gamma$ this canonical form $\ddagger$ is then given by

$$
\begin{aligned}
s^{3} & =\prod_{t=1}^{5}\left(t-T_{i}\right) \\
& =\lambda_{0}+\lambda_{1} t+\lambda_{2} t^{2}+\lambda_{3} t^{3}+\lambda_{4} t^{4}+t^{5} .
\end{aligned}
$$

We will call the Riemann surface for this cyclic $(3,5)$-curve $\mathbf{T}_{4}$. This surface is made from three sheets of the complex plane. It has branch points of order 3 at $T_{1}, \ldots, T_{5}, T_{6}=\infty$ and so there is just one infinite point. The local co-ordinate near $t=\infty$ is then $t=1 / \xi^{3}$. It follows that each sheet of $\mathbf{T}_{4}$ has branch cuts along the closed intervals

$$
\left[T_{1}, T_{2}\right], \quad\left[T_{2}, T_{3}\right], \quad\left[T_{4}, T_{5}\right], \quad\left[T_{5}, \infty\right]
$$

and is regular elsewhere. We note that if $P_{i}<P_{6}$ for $i \leq 5$, then $T_{i}>0$ for $i \leq 5$.

The three sheets are then connected in the same way as the Riemann surface $\Gamma$, replacing $P_{i}$ by $T_{i}$ for $i=1, \ldots, 5$ and $P_{6}$ by $\infty$.

The Weierstrass non-gap sequence for this cyclic $(3,5)$-curve is the set of all positive integers expressible as sums

$$
3 \alpha_{i}+5 \beta_{i}=W N G_{i}
$$

for non-negative integers $\alpha_{i}, \beta_{i}$. These numbers are:

$$
\{0,3,5,6,8, \ldots\} \text {. }
$$

$\ddagger$ We use the notation $\lambda_{i}$ for the moduli of the curve, following e.g. [15]. We should emphasise that these bear no direct relation to the function $\lambda(p)$ used above. 
Only the first $g$ terms of this are of interest here; all integers $\geq 2 g$ are trivially members of the sequence. The complement of this set is the Weierstrass gap sequence; here it is given by

$$
W G=\left\{\gamma_{4}, \gamma_{3}, \gamma_{2}, \gamma_{1}\right\}=\{1,2,4,7\} .
$$

Following [15], we can now define a set of holomorphic differentials on $\mathbf{T}_{4}$ by

$$
\mathrm{d} \mathbf{u}^{\mathrm{T}}(t, s)=\mathcal{U}^{\mathrm{T}} \frac{\mathrm{d} t}{f_{y}}=\left\{\mathcal{U}_{1}, \mathcal{U}_{2}, \mathcal{U}_{3}, \mathcal{U}_{4}\right\} \frac{\mathrm{d} t}{3 s^{2}},
$$

where $\mathcal{U}_{i}=t^{\alpha_{i}} s^{\beta_{i}}$ and the exponents $\alpha_{i}, \beta_{i}$ are as above. The analyticity of these differentials follows from direct expansion in terms of the local parameters at branch points. Solving equation (23) for $\alpha_{i}, \beta_{i} \in \mathbb{Z}$, as shown in table 1 , we see

$$
\mathrm{d} \mathbf{u}^{\mathrm{T}}=\left(1, t, s, t^{2}\right) \frac{\mathrm{d} t}{3 s^{2}} .
$$

\begin{tabular}{|c|c|c|c|}
\hline $\mathcal{U}_{i}$ & $W N G_{i}$ & $\alpha_{i}$ & $\beta_{i}$ \\
\hline $\mathcal{U}_{1}$ & 0 & 0 & 0 \\
\hline $\mathcal{U}_{2}$ & 3 & 1 & 0 \\
\hline $\mathcal{U}_{3}$ & 5 & 0 & 1 \\
\hline $\mathcal{U}_{4}$ & 6 & 2 & 0 \\
\hline
\end{tabular}

Table 1. A list of positive integers $P$ and $Q$ satisfying

$$
3 \alpha_{i}+5 \beta_{i}=W N G_{i}
$$

where $W N G_{i}$ is the $i$ th Weierstrass non-gap number.

These differentials may be re-expressed in terms of $p$ and $y$, to construct a set of holomorphic differentials on the original curve, but instead we will work with the canonical form of the curve, and transform the integral (13) into the variables $t$ and $s$. The integrand $(\varphi(p)-1) \mathrm{d} p(14)$ becomes

$$
\begin{aligned}
\varphi(p) \mathrm{d} p & =\left(\frac{\prod_{i=1}^{4}\left[\left(P_{6}-\hat{p}_{i}\right) t-1\right]}{\left\{\prod_{i=1}^{6}\left[\left(P_{6}-P_{i}\right) t-1\right]\right\}^{2 / 3}}\right) \frac{\mathrm{d} t}{t^{2}} \\
& =3 K^{2}\left(\frac{1+A_{1} t+A_{2} t^{2}+A_{3} t^{3}+A_{4} t^{4}}{\left[t^{5}+\lambda_{4} t^{4}+\lambda_{3} t^{3}+\lambda_{2} t^{2}+\lambda_{1} t+\lambda_{0}\right]^{2 / 3}}\right) \frac{\mathrm{d} t}{3 t^{2}} \\
& =3 K^{2}\left(\frac{1}{t^{2}}+\frac{A_{1}}{t}+A_{2}+A_{3} t+A_{4} t^{2}\right) \frac{\mathrm{d} t}{3 s^{2}}
\end{aligned}
$$

where $A_{i}$ are constants, and $K$ is defined as above.

We note that we can write the constant $A_{1}$ in terms of the curve moduli, $\lambda_{i}$, as follows. Evaluating (25) explicitly we find

$$
A_{1}=-\sum_{i=1}^{4}\left(P_{6}-\hat{p}_{i}\right)
$$


and

$$
\lambda_{0}=\frac{1}{3} K^{3 / 2}, \quad \lambda_{1}=\frac{\sum_{i=1}^{5}\left(P_{6}-P_{i}\right)}{K^{3 / 2}} .
$$

If we now use identity (16), which relates the branch points $P_{i}$ to the $\hat{p}_{j}$, we see

$$
\begin{aligned}
A_{1} & =-\left(4 P_{6}-\sum_{i=1}^{4} \hat{p}_{i}\right)=-\left(4 P_{6}-\frac{2}{3} \sum_{i=1}^{6} P_{i}\right) \\
& =-\frac{2}{3}\left(5 P_{6}-\sum_{i=1}^{5} P_{i}\right)=-\frac{2}{3} \sum_{i=1}^{5}\left(P_{6}-P_{i}\right)=\frac{2}{3} \frac{\lambda_{1}}{\lambda_{0}} .
\end{aligned}
$$

It follows that

$$
\varphi(t) \mathrm{d} t=k\left(A_{2}+A_{3} t+A_{4} t^{2}\right) \frac{\mathrm{d} t}{3 s^{2}}+k\left(\frac{1}{t^{2}}+\frac{2}{3} \frac{\lambda_{1}}{\lambda_{0}} \frac{1}{t}\right) \frac{\mathrm{d} t}{3 s^{2}},
$$

where the first term is a sum of holomorphic differentials and the second term is a second kind differential.

To identify functions on the surface $\mathbf{T}_{4}$, we first define the period matrices:

$$
2 \omega_{i j}=\oint_{\mathfrak{a}_{j}} \mathrm{~d} u_{i}, \quad 2 \omega_{i j}^{\prime}=\oint_{\mathfrak{b}_{j}} \mathrm{~d} u_{i}
$$

where $\omega$ and $\omega^{\prime}$ are $4 \times 4$ matrices.

The lattice of points generated by these periods is given by

$$
\Lambda=\left\{2 \mathbf{m} \omega+2 \mathbf{n} \omega^{\prime}: \mathbf{m}, \mathbf{n} \in \mathbb{Z}^{4}\right\} .
$$

We define Abelian functions on $\mathbb{C}^{4}$ as meromorphic functions which are invariant under translations by this period lattice $\Lambda$; that is, they satisfy

$$
f(p+2 \mathbf{n} \omega+2 \mathbf{m} \omega)=f(p)
$$

for $\mathbf{n}, \mathbf{m} \in \mathbb{Z}$. We now define the Jacobian of $\mathbf{T}_{4}$ by $\operatorname{Jac}\left(\mathbf{T}_{4}\right)=\mathbb{C}^{4} / \Lambda$. As in the hyperelliptic cases, we can map $\mathbf{T}_{4}$ into $\operatorname{Jac}\left(\mathbf{T}_{4}\right)$ using the Abel map. For any point $t$ and base point $t_{a} \in \mathbf{T}_{4}$ this is given by

$$
\begin{aligned}
\mathfrak{A}(t) & =\int_{t_{a}}^{t} \mathrm{~d} \mathbf{u}\left(t^{\prime}\right) \quad \bmod (\Lambda) \\
& =\mathbf{u}(t)
\end{aligned}
$$

The map $\mathfrak{A}(t)$ forms a 1 dimensional image of the $\mathbf{T}_{4}$, a subset of the 4 dimensional $\operatorname{Jac}\left(\mathbf{T}_{4}\right)$. We denote this one dimensional stratum of $\operatorname{Jac}\left(\mathbf{T}_{4}\right)$ by

$$
\Theta_{1}=\left\{\mathbf{u}: \mathbf{u}=\int_{t_{a}}^{t} \mathrm{~d} \mathbf{u} \bmod (\Lambda)\right\} .
$$

Henceforth we will always choose the base point $t_{a}=\infty$. Since $\lambda(t)$ is given by a single integral with respect to one parameter, a point $(t, s) \in \mathbf{T}_{4}$, it makes sense to rewrite the integral (25) as an integral on the one-dimensional stratum $\Theta_{1}$ of $\operatorname{Jac}\left(\mathbf{T}_{4}\right)$. Thus, we need to understand how meromorphic functions on $\mathbf{T}_{4}$ correspond to the restrictions of Abelian functions to this subspace of the Jacobi variety. Similar such problems of inverting meromorphic differentials on lower-dimensional strata of the Jacobi variety of a curve have been studied for example, by Alber and Fedorov, [20], and Enolski, Pronine and Richter, [21]. 


\section{Abelian differentials and the sigma function}

It is possible to construct the correspondence between meromorphic functions on $\mathbf{T}_{4}$ and the restrictions of Abelian functions on $\operatorname{Jac}\left(\mathbf{T}_{4}\right)$ to $\Theta_{1}$, using the Kleinian $\sigma$ function. Key to this construction was the definition of the associated second kind differentials and the set of normalized holomorphic differentials. Thus we will begin by constructing a set of associated second-kind differentials on the Riemann surface $\mathbf{T}_{4}$ and then recall the main properties of the normalized differentials.

\subsection{Differentials}

We recall that $\mathbf{T}_{4}$ has the set of holomorphic differentials (24):

$$
\begin{aligned}
\mathrm{d} \mathbf{u}^{\mathrm{T}} & =\mathcal{U}^{\mathrm{T}} \frac{\mathrm{d} t}{3 s^{2}}=\left(\mathcal{U}_{1}, \mathcal{U}_{2}, \mathcal{U}_{3}, \mathcal{U}_{4}\right) \frac{\mathrm{d} t}{3 s^{2}} \\
& =\left(1, t, s, t^{2}\right) \frac{\mathrm{d} t}{3 s^{2}} .
\end{aligned}
$$

To evaluate a set of associated second kind differentials

$$
\mathrm{d} \mathbf{r}^{\mathrm{T}}=\left(\mathcal{R}_{1}, \mathcal{R}_{2}, \mathcal{R}_{3}, \mathcal{R}_{4}\right) \frac{\mathrm{d} t}{3 s^{2}} .
$$

we use the procedure described in [15] and [16].

Klein's fundamental second kind 2-form, $\mathrm{d} \Omega(t, z)$ on $\mathbf{T}_{4}$ is defined as the unique 2-form, depending symmetrically on two distinct points $(t, s)$ and $(z, w)$ on $\mathbf{T}_{4}$ :

$$
\begin{aligned}
& s^{3}=t^{5}+\lambda_{4} t^{4}+\lambda_{3} t^{3}+\lambda_{2} t^{2}+\lambda_{1} t+\lambda_{0}, \\
& w^{3}=z^{5}+\lambda_{4} z^{4}+\lambda_{3} z^{3}+\lambda_{2} z^{2}+\lambda_{1} z+\lambda_{0} \\
& (t, s) \neq(z, w),
\end{aligned}
$$

which satisfies:

$$
\mathrm{d} \Omega(t, z) \simeq\left(\frac{1}{(t-z)^{2}}+\mathrm{O}(1)\right) \mathrm{d} t \mathrm{~d} z
$$

with no singularities except on the diagonal $(t, s)=(z, w)$.

It may be constructed by setting

$$
\mathrm{d} \Omega(t, z)=\frac{\mathrm{d}}{\mathrm{d} z}\left(\frac{\Psi^{\mathrm{T}}(z, w) \Phi(t, s)}{t-z}\right) \mathrm{d} z \frac{\mathrm{d} t}{3 s^{2}}+\mathcal{R}^{\mathrm{T}}(z, w) \mathcal{U}(t, s) \frac{\mathrm{d} z}{3 w^{2}} \frac{\mathrm{d} t}{3 s^{2}}
$$

and where

$$
\Psi^{\mathrm{T}}(z, w)=\left(1, w, w^{2}\right), \quad \Phi^{\mathrm{T}}(t, s)=\left(s^{2}, s, 1\right) .
$$

To identify the unknown polynomials $\mathcal{R}^{\mathrm{T}}(t, s)=\left(\mathcal{R}_{1}, \mathcal{R}_{2}, \mathcal{R}_{3}, \mathcal{R}_{4}\right)$, we impose the symmetry condition

$$
\mathrm{d} \Omega(t, z)-\mathrm{d} \Omega(z, t)=0 .
$$


We find:

$$
\begin{aligned}
\mathrm{d} \Omega(t, z)= & \frac{\mathrm{d}}{\mathrm{d} z}\left(\frac{s^{2}+s w+w^{2}}{t-z}\right) \frac{\mathrm{d} z \mathrm{~d} t}{3 s^{2}} \\
& +\left(\mathcal{R}_{1}(z, w)+t \mathcal{R}_{2}(z, w)+s \mathcal{R}_{3}(z, w)+t^{2} \mathcal{R}_{4}(z, w)\right) \frac{\mathrm{d} z}{3 w^{2}} \frac{\mathrm{d} t}{3 s^{2}} \\
= & {\left[\frac{s w_{z}+2 w w_{z}}{t-z}-\frac{s^{2}+s w+w^{2}}{(t-z)^{2}}\right] \frac{\mathrm{d} z \mathrm{~d} t}{3 s^{2}} } \\
& +\left(\mathcal{R}_{1}(z, w)+t \mathcal{R}_{2}(z, w)+s \mathcal{R}_{3}(z, w)+t^{2} \mathcal{R}_{4}(z, w)\right) \frac{\mathrm{d} z}{3 w^{2}} \frac{\mathrm{d} t}{3 s^{2}} .
\end{aligned}
$$

If we multiply this by

$$
\frac{3 s^{2}}{\mathrm{~d} t} \frac{3 w^{2}}{\mathrm{~d} z}
$$

and then set

$$
Q_{1}(t, z)=\frac{3 w^{2} s w_{z}+6 w^{3} w_{z}}{t-z}-\frac{3 w^{2} s^{2}+3 w^{3} s+3 w^{4}}{(t-z)^{2}}
$$

and

$$
Q_{2}(t, z)=\mathcal{R}_{1}(z, w)+t \mathcal{R}_{2}(z, w)+s \mathcal{R}_{3}(z, w)+t^{2} \mathcal{R}_{4}(z, w),
$$

then the symmetry condition is equivalent to

$$
Q_{1}(t, z)-Q_{1}(z, t)=Q_{2}(z, t)-Q_{2}(t, z) .
$$

We simplify the left hand side of (32) using

$$
w^{3}=z^{5}+\lambda_{4} z^{4}+\lambda_{3} z^{3}+\lambda_{2} z^{2}+\lambda_{1} z+\lambda_{0},
$$

and

$$
3 w^{2} w_{z}=\frac{\mathrm{d}}{\mathrm{d} z}\left(w^{3}\right)=5 z^{4}+4 \lambda_{4} z^{3}+3 \lambda_{3} z^{2}+2 \lambda_{2} z+\lambda_{1} .
$$

This gives

$$
\begin{aligned}
Q_{1}(t, z)= & {\left[s \frac{\mathrm{d}}{\mathrm{d} z}\left(w^{3}\right)+2 w^{2} \frac{\mathrm{d}}{\mathrm{d} z}\left(w^{3}\right)\right] \frac{1}{(t-z)} } \\
& +\left[3 w^{2} s^{2}+3 s w\left(w^{3}\right)+3 w^{2}\left(w^{3}\right)\right] \frac{1}{(t-z)^{2}} \\
= & \frac{s+2 w^{2}}{t-z}\left(5 z^{4}+4 \lambda_{4} z^{3}+3 \lambda_{3} z^{2}+2 \lambda_{2} z+\lambda_{1}\right)+\frac{3 w^{2} s^{2}}{(t-z)^{2}} \\
& +3 \frac{s+w^{2}}{(t-z)^{2}}\left(z^{5}+\lambda_{4} z^{4}+\lambda_{3} z^{3}+\lambda_{2} z^{2}+\lambda_{1} z+\lambda_{0}\right)
\end{aligned}
$$

with $Q_{1}(z, t)$ evaluated in a similar way. If we now expand the expression $\left(Q_{1}(t, z)-\right.$ $\left.Q_{1}(z, t)\right)$ and then rearrange, we obtain

$$
\begin{aligned}
& Q_{1}(t, z)-Q_{1}(z, t)= \\
& \quad\left(4 t^{2} z+3 t \lambda_{3}+2 t z \lambda_{4}+\lambda_{2}+t z^{2} \lambda_{5}-2 z^{3} \lambda_{5}-z^{2} \lambda_{4}+5 t^{2} \lambda_{4}+7 t^{3} \lambda_{5}\right) s \\
& -\left(4 z^{2} t+3 z \lambda_{3}+2 t z \lambda_{4}+\lambda_{2}+t z^{2} \lambda_{5}-2 t^{3} \lambda_{5}-t^{2} \lambda_{4}+5 z^{2} \lambda_{4}+7 z^{3} \lambda_{5}\right) w
\end{aligned}
$$


Recalling that the right hand side of $(32)$ is

$$
\begin{aligned}
Q_{2}(z, t)-Q_{2}(t, z)= & \left(\mathcal{R}_{1}(t, s)+z \mathcal{R}_{2}(t, s)+w \mathcal{R}_{3}(t, s)+z^{2} \mathcal{R}_{4}(t, s)\right) \\
& -\left(\mathcal{R}_{1}(z, w)+t \mathcal{R}_{2}(z, w)+s \mathcal{R}_{3}(z, w)+t^{2} \mathcal{R}_{4}(z, w)\right),
\end{aligned}
$$

we can now evaluate the polynomials $\mathcal{R}_{i}(t, s)$ by matching coefficients of $s$ and $w$. We note that the $\mathcal{R}_{i}(t, s)$ are not defined uniquely, but one such set is given by

$$
\begin{aligned}
& \mathcal{R}_{1}(t, s)=s t\left(3 \lambda_{3}+7 t^{2}+5 t \lambda_{4}\right), \\
& \mathcal{R}_{2}(t, s)=2 s t\left(2 t+\lambda_{4}\right), \\
& \mathcal{R}_{3}(t, s)=2 t^{3}+t^{2} \lambda_{4}-\lambda_{2}, \\
& \mathcal{R}_{4}(t, s)=s t
\end{aligned}
$$

The second kind differentials dr associated to the set of first kind differentials du are then given by

$$
\mathrm{d} \mathbf{r}^{\mathrm{T}}=\left(\mathcal{R}_{1}, \mathcal{R}_{2}, \mathcal{R}_{3}, \mathcal{R}_{4}\right) \frac{\mathrm{d} t}{3 s^{2}}
$$

we can now define the corresponding two $4 \times 4$ period matrices $\eta$ and $\eta^{\prime}$ :

$$
2 \eta_{i j}=-\oint_{\mathfrak{a}_{j}} \mathrm{~d} r_{i}, \quad 2 \eta_{i j}^{\prime}=-\oint_{\mathfrak{b}_{j}} \mathrm{~d} r_{i}, \quad(i, j=1, \ldots, g) .
$$

By construction, and the use of Riemann's bilinear identity, these period matrices $\eta, \eta^{\prime}$ and $\omega, \omega^{\prime}$ must satisfy the generalized Legendre relation; if the $2 g \times 2 g$ matrix $\mathfrak{M}$ is defined by

$$
\mathfrak{M}=\left(\begin{array}{cc}
\omega & \omega^{\prime} \\
\eta & \eta^{\prime}
\end{array}\right)
$$

then:

$$
\mathfrak{M}\left(\begin{array}{cc}
0 & -1_{g} \\
1_{g} & 0
\end{array}\right) \mathfrak{M}^{\mathrm{T}}=-\frac{i \pi}{2}\left(\begin{array}{cc}
0 & -1_{g} \\
1_{g} & 0
\end{array}\right)
$$

and so $\mathfrak{M}$ belongs, up to a factor of $\sqrt{-\frac{i \pi}{2}}$, to the $\operatorname{Symplectic}$ group $\operatorname{Sp}(8, \mathbb{C})$ (see, e.g. [22], p. 37].

\subsection{The $\sigma$ function}

If we introduce the normalized holomorphic differentials $\mathrm{d} \mathbf{v}$ on $\mathbf{T}_{4}$ by setting

$$
\oint_{\mathfrak{a}_{i}} \mathrm{~d} v_{j}=\delta_{i, j}, \quad i, j=1, \ldots, 4
$$

then their periods around the $\mathfrak{b}$ cycles are given by

$$
\oint_{\mathfrak{b}_{i}} \mathrm{~d} v_{j}=\left(\omega^{-1} \omega^{\prime}\right)_{i, j}=\tau_{i, j}, \quad i, j=1, \ldots, 4,
$$

where, as usual, the matrix $\tau$ must be symmetric, with positive definite imaginary part.

Following [19], we can now define the Kleinian $\sigma$ function on $\mathbf{T}_{4}$ : 
Definition 3.1 Let $t_{a}$ be any regular point on the Riemann surface $\mathbf{T}_{4}$, and let $\left\{t_{1}, \ldots, t_{4}\right\} \in\left(\mathbf{T}_{4}\right)^{4}$; the Abel map of the divisor $\left(t_{1}+t_{2}+t_{3}+t_{4}\right)$ is defined by:

$$
\mathbf{u}=\sum_{i=1}^{4} \int_{t_{a}}^{t_{i}} \mathrm{~d} \mathbf{u} .
$$

Then the fundamental Abelian $\sigma$-function on $\mathbb{C}^{4}$, the covering space of $\operatorname{Jac}\left(\mathbf{T}_{4}\right)$, is given by

$$
\begin{aligned}
\sigma(\mathbf{u} ; \mathfrak{M})= & \frac{1}{\sqrt[4]{\mathrm{D}(v)}} \frac{\pi}{\sqrt{\operatorname{det}(\omega)}} \exp \left(\frac{1}{2} \mathbf{u}^{\mathrm{T}} \eta \omega^{-1} \mathbf{u}\right) \\
& \sum_{\mathbf{m} \in \mathbb{Z}^{4}} \exp \left(i \pi\left(\mathbf{m}^{T} \tau \mathbf{m}+2 \mathbf{m}^{T}\left((2 \omega)^{-1} \mathbf{u}-\Delta_{t_{a}}\right)\right)\right.
\end{aligned}
$$

where $\mathrm{D}(v)$ is the discriminant of the curve $\mathbf{T}_{4}$

$$
\mathrm{D}(v)=\prod_{1 \leq i<j \leq 5}\left(T_{i}-T_{j}\right)
$$

and $\Delta_{t_{a}}$ is the Riemann constant with base point $t_{a}$; if we fix $t_{a}=\infty$, and choose the homology basis as in [18] then the corresponding Riemann constant $\Delta_{\infty}$ was shown there to be

$$
\Delta_{\infty}=\left(\frac{1}{2}, \frac{1}{2}, \frac{1}{2}, \frac{1}{2}\right)^{T}
$$

The fundamental properties of $\sigma$ are:

- it is an entire function on $\mathbb{C}^{4}$, the covering space of $\operatorname{Jac}\left(\mathbf{T}_{4}\right)$,

- it is quasi-periodic:

$$
\begin{aligned}
& \sigma\left(\mathbf{u}+2 \omega \mathbf{k}+2 \omega^{\prime} \mathbf{k}^{\prime} ; \mathfrak{M}\right)= \\
& \quad \exp \left\{2\left(\eta \mathbf{k}+\eta^{\prime} \mathbf{k}^{\prime}\right)^{T}\left(\mathbf{u}+\omega \mathbf{k}+\omega^{\prime} \mathbf{k}^{\prime}\right)\right\} \sigma(\mathbf{u} ; \mathfrak{M})
\end{aligned}
$$

- it is invariant under changes in the basis of cycles - it is a modular invariant:

$$
\sigma(\mathbf{u} ; \gamma \mathfrak{M})=\sigma(\mathbf{u} ; \mathfrak{M}), \gamma \in \operatorname{Sp}(2 g, \mathbb{Z})
$$

- the first term of the of the $\sigma$-series is the Schur-Weierstrass polynomial which is defined as follows. If $e_{k}$ is the elementary symmetric function of weight $k$ with respect to the variables $z_{1}, \ldots, z_{g}$, then the $\operatorname{determinant} \operatorname{det}\left(e_{g+j-2 k+1}\right)_{j, k=1, \ldots, g}$, can necessarily be expressed as a polynomial in terms of Newton polynomials $p_{2 k-1}=z_{1}^{2 k-1}+\ldots+z_{g}^{2 k-1}, k=1, \ldots, g$. The substitution $p_{2 k-1}=u_{k}, k=1, \ldots, g$ defines the required Schur-Weierstrass polynomial in $\operatorname{Jac}\left(\mathbf{T}_{4}\right)$; for the curve $\mathbf{T}_{4}$ this polynomial has weight 8 in the Sato-Weierstrass grading, where we assign weights:

$$
\left|u_{1}\right|=7, \quad\left|u_{2}\right|=4, \quad\left|u_{3}\right|=2, \quad\left|u_{4}\right|=1 .
$$

- the higher order terms in the Taylor expansion of $\sigma$ with respect to $\left(u_{1}, u_{2}, u_{3}, u_{4}\right)$ are also all isobaric polynomials of weight 8 in these variables and the curve moduli $\left(\lambda_{0}, \lambda_{1}, \lambda_{2}, \lambda_{3}, \lambda_{4}\right)$, where the weights of the moduli are assigned as follows:

$$
\left|\lambda_{0}\right|=-15, \quad\left|\lambda_{1}\right|=-12, \quad\left|\lambda_{2}\right|=-9, \quad\left|\lambda_{3}\right|=-6, \quad\left|\lambda_{4}\right|=-3 .
$$


This is used to define a higher genus analogue of the $\zeta$ function and Weierstrass' $\wp$ function. We have, analogously to the elliptic case,

and

$$
\zeta_{i}(\mathbf{u})=\frac{\partial}{\partial u_{i}}[\log \sigma(\mathbf{u})]=\frac{\sigma_{i}}{\sigma}(\mathbf{u}), \quad(i=1, \ldots, 4)
$$

$$
\wp_{i j}(\mathbf{u})=-\frac{\partial^{2}}{\partial u_{i} \partial u_{j}}[\log \sigma(\mathbf{u})]=-\frac{\sigma_{i j}}{\sigma}(\mathbf{u})+\frac{\sigma_{i} \sigma_{j}}{\sigma^{2}}(\mathbf{u}), \quad(i, j=1, \ldots, 4)
$$

where we denote:

$$
\sigma_{i}=\frac{\partial \sigma}{\partial u_{i}}, \quad \sigma_{i j}=\frac{\partial^{2} \sigma}{\partial u_{j} \partial u_{i}}, \cdots
$$

Higher order logarithmic derivatives are written, for example,

$$
\wp_{i j k}(\mathbf{u})=-\frac{\partial^{3}}{\partial u_{i} \partial u_{j} \partial u_{k}}[\log \sigma(\mathbf{u})], \quad(i, j, k=1, \ldots, 4) .
$$

The periodicity properties of these functions are as follows:

(i) $\left.\zeta_{i}\left(\mathbf{u}+2 \omega \mathbf{m}+2 \omega^{\prime} \mathbf{m}^{\prime}\right)\right)=\zeta_{i}(\mathbf{u})+2\left(\eta \mathbf{m}+\eta^{\prime} \mathbf{m}^{\prime}\right)_{i} \quad(i, j=1, \ldots, 4)$.

where the subscript $i$ on the last term denotes the $i$ th component of this vector;

(ii) $\left.\wp_{i j}\left(\mathbf{u}+2 \omega \mathbf{m}+2 \omega^{\prime} \mathbf{m}^{\prime}\right)\right)=\wp_{i j}(\mathbf{u}) \quad(i, j=1, \ldots, 4)$.

Thus the Kleinian $\wp_{i j}$ functions and all their derivatives are Abelian functions on $\operatorname{Jac}\left(\mathbf{T}_{4}\right)$.

3.3. Genus 4 Trigonal curve: Jacobi's inversion theorem and some relations between the $\wp_{i j k l}$

We begin by rewriting Klein's theorem (Thm. (3.4) in [15]), for the case of the curve $\mathbf{T}_{4}$.

Theorem 3.1 For arbitrary distinct $(t, s)$, and base point $\left(t_{a}, s\left(t_{a}\right)\right)$ on $\mathbf{T}_{4}$ and an arbitrary set of $g=4$ distinct points $\left\{\left(t_{1}, s_{1}\right), \ldots,\left(t_{4}, s_{4}\right)\right\} \in\left(\mathbf{T}_{4}\right)$ it follows that

$$
\begin{gathered}
\sum_{i, j=1}^{4} \wp_{i, j}\left(\int_{t_{a}}^{t} \mathrm{~d} \mathbf{u}-\sum_{k=1}^{4} \int_{t_{a}}^{t_{k}} \mathrm{~d} \mathbf{u}\right) \mathcal{U}_{i}(t, s) \mathcal{U}_{j-1}\left(t_{r}, s_{r}\right)=\frac{F\left(t, s ; t_{r}, s_{r}\right)}{\left(t-t_{r}\right)^{2}} \quad r=1, \ldots, 4(38 \\
\mathcal{U}^{\mathrm{T}}(t, s)=\left(1, t, s, t^{2}\right)
\end{gathered}
$$

and $F$ is the symmetric function

$$
\begin{aligned}
F\left(t, s ; t_{r}, s_{r}\right)= & 3 s_{r}^{2} s^{2}+ \\
& {\left[2 t_{r}^{3} t^{2}+t_{r}^{4} t+3 \mu_{0}+\mu_{1}\left(2 t_{r}+t\right)+\mu_{2}\left(t_{r}^{2}+2 t t_{r}\right)+\mu_{3}\left(3 t_{r}^{2} t\right)+\mu_{4}\left(2 t_{r}^{3} t+t^{2} t_{r}^{2}\right)\right] s } \\
& {\left[2 t^{3} t_{r}^{2}+t^{4} t_{r}+3 \mu_{0}+\mu_{1}\left(2 t+t_{r}\right)+\mu_{2}\left(t^{2}+2 t t_{r}\right)+\mu_{3}\left(3 t^{2} t_{r}\right)+\mu_{4}\left(2 t^{3} t_{r}+t^{2} t_{r}^{2}\right)\right] s_{r} }
\end{aligned}
$$

appearing in the numerator of the second kind fundamental 2-form:

$$
\frac{F\left(t, s ; t_{r}, s_{r}\right)}{\left(t-t_{r}\right)^{2}} \frac{\mathrm{d} t}{\left(3 s^{2}\right)} \frac{\mathrm{d} t^{r}}{\left(3 s_{r}^{2}\right)}=\mathrm{d} \Omega\left(t, s ; t_{r}, s_{r}\right)
$$


This result allows us to write down the Jacobi inversion formula on $\mathbf{T}_{4}$ explicitly, and also to find some PDE satisfied by the $\wp$ derivatives on $\Theta_{4}$. To do this we follow the procedure outlined in $[15]$ for a $(3,4)$ curve.

We fix the base point $t_{a}$ of the Abel map at infinity. We then let $t \rightarrow \infty$. Expanding equation (38) in the local co-ordinate $t=1 / \xi^{3}$ gives the following Taylor series expansion:

$$
R H S=\left(-\frac{1}{3} \frac{t_{r}}{s_{r}}\right)+\left(-\frac{\lambda_{4}}{3} \frac{t_{r}^{2}}{s_{r}^{2}}-\frac{2}{3} \frac{t_{r}^{3}}{s_{r}^{2}}\right) \xi-\xi^{2}+O\left(\xi^{3}\right)
$$

and

$$
\begin{aligned}
L H S= & \left(-\frac{\wp_{44}}{3} \frac{t_{r}^{2}}{s_{r}^{2}}-\frac{\wp_{34}}{3} \frac{1}{s_{r}}-\frac{\wp_{24}}{3} \frac{t_{r}}{s_{r}^{2}}-\frac{\wp_{14}}{3} \frac{1}{s_{r}^{2}}\right)+ \\
& -\left[\frac{\wp_{344}+\wp_{33}}{3 s_{r}}+\frac{\wp_{13}+\wp_{144}+t_{r}\left(\wp_{23}+\wp_{244}\right)+t_{r}^{2}\left(\wp_{34}+\wp_{444}\right)}{3 s_{r}^{2}}\right] \xi \\
& +\left[-\left(2 \wp_{334}-\wp_{3444}-\wp_{334}\right) \frac{1}{6 s_{r}}-\left(3 \wp_{134}+t_{r}\left(2 \wp_{234}+\wp_{2444}+\wp_{234}\right)\right.\right. \\
& \left.\left.-t_{r}^{2}\left(2 \wp_{344}+\wp_{4444}-\wp_{344}\right)\right) \frac{1}{6 s_{r}^{2}}\right] \xi^{2}+O\left(\xi^{3}\right)
\end{aligned}
$$

If we multiply both sides by $3 s_{r}^{2}$ and subtract the right hand side from the left, then the coefficient of $\xi^{i}, C_{i}$, is:

$$
\begin{aligned}
C_{0}= & \wp_{14}+\wp_{24} t_{r}+\wp_{34} s_{r}+\wp_{44} t_{r}^{2}-t_{r} s_{r} ; \\
C_{1}= & \left(\wp_{13}+\wp_{144}\right)+\left(\wp_{23}+\wp_{244}\right) t_{r} \\
& +\left(\wp_{33}+\wp_{344}\right) s_{r}+\left(\wp_{34}+\wp_{444}-\lambda_{4}\right) t_{r}^{2}-2 t_{r}^{3} ; \\
C_{2}= & -\frac{1}{2}\left[\wp_{1444}+3 \wp_{134}+\left(3 \wp_{234}+\wp_{2444}\right) t_{r}\right. \\
& \left.+\left(3 \wp_{334}+\wp_{3444}\right) s_{r}+\left(\wp_{4444}+3 \wp_{344}\right) t_{r}^{2}\right]-1 .
\end{aligned}
$$

It follows that $C_{i}$ must be zero for any $\mathbf{u} \in \Theta_{4}$ and some $\left(t_{r}, s_{r}\right) \in \mathbf{T}_{4}$.

In the hyperelliptic case, the first term, analogous to $C_{0}$ here, defines the inversion equation - there it is a polynomial of order $g$ in the unknown $t_{r}$. Here, however, equation (39), however, contains both $t_{r}$ and $s_{r}$ and so we simplify this further by eliminating $s_{r}$. Evaluating the resultant of $C_{0}$ and $C_{1}$ with respect to $s_{r}$ gives the quartic

$$
\begin{aligned}
D_{01}= & 2 t_{r}^{4}+\left(\lambda_{4}-\wp_{444}-3 \wp_{34}\right) t_{r}^{3} \\
& +\left(-\wp_{34} \lambda_{4}-\wp_{44} \wp_{33}+\wp_{444} \wp_{34}+\wp_{34}^{2}-\wp_{244}-\wp_{23}-\wp_{44} \wp_{344}\right) t_{r}^{2} \\
& +\left(-\wp_{13}-\wp_{144}+\wp_{244} \wp_{34}+\wp_{23} \wp_{34}-\wp_{24} \wp_{344}-\wp_{24} \wp_{33}\right) t_{r} \\
& +\wp_{13} \wp_{34}-\wp_{14} \wp_{344}-\wp_{14} \wp_{33}+\wp_{144} \wp_{34} .
\end{aligned}
$$

This forms the key equation in Jacobi's inversion theorem for the curve $\mathbf{T}_{4}$. For each of the four roots $\left\{t_{1}, \ldots t_{4}\right\}$ of this equation, the corresponding point $s_{i}$ can be found from $C_{0}=0$. The $g=4$ points $\left(t_{i}, s_{i}\right) \in \mathbf{T}_{4}$ form the Abel preimage of $\mathbf{u}$.

If we similarly eliminate $s_{r}$ from the pair of equations $C_{0}$ and $C_{2}$ then we obtain $D_{02}=\quad\left(3 \wp_{44}^{2}-\frac{3}{2} \wp_{344}-\frac{1}{2} \wp_{4444}\right) t_{r}^{4}+\left(6 \wp_{44} \wp_{24}-\frac{3}{2} \wp_{334} \wp_{44}-\frac{1}{2} \wp_{2444}\right.$ 


$$
\begin{aligned}
& \left.+\wp_{4444} \wp_{34}+3 \wp_{344} \wp_{34}-\frac{3}{2} \wp_{234}-\frac{1}{2} \wp_{3444} \wp_{44}\right) t_{r}^{3} \\
& +\left(-\frac{3}{2} \wp_{344} \wp_{34}^{2}+3 \wp_{234} \wp_{34}-\frac{1}{2} \wp_{3444} \wp_{24}+\wp_{2444} \wp_{34}-\frac{1}{2} \wp_{4444} \wp_{34}^{2}+3 \wp_{24}^{2}\right. \\
& \left.-\frac{3}{2} \wp_{134}+\frac{1}{2} \wp_{3444} \wp_{34} \wp_{44}+6 \wp_{44} \wp_{14}+\frac{3}{2} \wp_{334} \wp_{34} \wp_{44}-\frac{3}{2} \wp_{334} \wp_{24}-\frac{1}{2} \wp_{1444}\right) t_{r}^{2} \\
& \left(-\frac{1}{2} \wp_{3444} \wp_{14}+\frac{1}{2} \wp_{3444} \wp_{34} \wp_{24}-\frac{3}{2} \wp_{334} \wp_{14}+3 \wp_{134} \wp_{34}+6 \wp_{14} \wp_{24}\right. \\
& \left.-\frac{1}{2} \wp_{2444} \wp_{34}^{2}-\frac{3}{2} \wp_{234} \wp_{34}^{2}+\frac{3}{2} \wp_{334} \wp_{34} \wp_{24}+\wp_{1444} \wp_{34}\right) t_{r} \\
& +\left(3 \wp_{14}^{2}+\frac{3}{2} \wp_{334} \wp_{34} \wp_{14}-\frac{3}{2} \wp_{134} \wp_{34}^{2}+\frac{1}{2} \wp_{3444} \wp_{34} \wp_{14}-\frac{1}{2} \wp_{1444} \wp_{34}^{2}\right)
\end{aligned}
$$

another quartic in $t_{r}$ which must have the same roots $\left\{t_{i}\right\}$ as $D_{0,1}$. Multiplying $D_{01}$ by

$$
\left(3 \wp_{44}^{2}-\frac{3}{2} \wp_{344}-\frac{1}{2} \wp_{4444}\right)
$$

and subtracting this from $D_{0,2}$, we have a cubic equation in $z$. Since this must be satisfied by four distinct $t_{i} \in \mathbf{T}_{4}$ the coefficients in this cubic must equal zero. This allows us to rewrite the highest order $\wp$ derivative in each coefficient in terms of lower order derivatives. For example, from this pair of equations, $D_{0,1}$ and $D_{0,2}$, we obtain an expression for $\wp_{4444}$ from the coefficient of $z^{3}, \wp_{3444}$ from $z^{2}, \wp_{2444}$ from $z$ and $\wp_{1444}$ from the constant term. Simplifying these, we find, for example,

$\wp_{4444}=\frac{3}{\wp_{33}+\wp_{344}}\left(4 \wp_{34}^{2} \wp_{44}+4 \wp_{24} \wp_{34}+2 \wp_{44}^{2} \wp_{344}+2 \wp_{44}^{2} \wp_{33}-\wp_{344}^{2}-\wp_{344} \wp_{33}+4 \wp_{14}\right)$.

By looking at higher order terms of $\xi$ in the expansion of theorem 3.1 and eliminating $s_{r}$ and $t_{r}$ as shown above, we may obtain relations for more of the $\wp$ derivatives. It is not yet clear how a fundamental set of such relations might be constructed.

Theorem 3.2 (Jacobi inversion for genus 4 Trigonal curve) [[19], p 32] Let $\mathbf{T}_{4}$ be the genus 4 cyclic $(3,5)$-curve defined by

$$
s^{3}=t^{5}+\sum_{i=0}^{4} \lambda_{i} t^{i},
$$

let

$$
u_{i}=\sum_{k=1}^{4} \int_{\infty}^{t_{k}} \mathrm{~d} u_{i},
$$

where $\left(t_{1}+t_{2}+t_{3}+t_{4}\right)$ is a non-special divisor and $\mathrm{d} \mathbf{u}$ is the vector of holomorphic differentials.

The Abel preimage of the point $\mathbf{u} \in \mathbf{T}_{4}$ is then given by the set $\left\{\left(t_{1}, s_{1}\right), \ldots,\left(t_{4}, s_{4}\right)\right\} \in\left(\mathbf{T}_{4}\right)^{4}$, where $\left\{t_{1}, \ldots, t_{4}\right\}$ are the zeros of the polynomial

$$
\begin{aligned}
\mathcal{P}(t ; \mathbf{u})= & 2 t^{4}+\left(\lambda_{4}-\wp_{444}-3 \wp_{34}\right) t^{3} \\
& +\left(-\wp_{34} \lambda_{4}-\wp_{44} \wp_{33}+\wp_{444} \wp_{34}+\wp_{34}{ }^{2}-\wp_{244}-\wp_{23}-\wp_{44} \wp_{344}\right) t^{2}
\end{aligned}
$$




$$
\begin{aligned}
& +\left(-\wp_{13}-\wp_{144}+\wp_{244} \wp_{34}+\wp_{23} \wp_{34}-\wp_{24} \wp_{344}-\wp_{24} \wp_{33}\right) t \\
& +\wp_{13} \wp_{34}-\wp_{14} \wp_{344}-\wp_{14} \wp_{33}+\wp_{144} \wp_{34},
\end{aligned}
$$

and the pairs $\left.\left\{\left(t_{r}, s_{r}\right)\right\}\right|_{i=1} ^{4}$ each satisfy:

$$
\mathcal{Q}\left(t_{r}, s_{r} ; \mathbf{u}\right)=0
$$

where

$$
\mathcal{Q}\left(t_{r}, s_{r} ; \mathbf{u}\right)=\wp_{14}+\wp_{24} t_{r}+\wp_{34} s_{r}+\wp_{44} t_{r}^{2}-t_{r} s_{r}
$$

\subsection{Strata of the Jacobian and the inversion theorem on $\Theta_{1}$}

Consider $\left(\mathbf{T}_{4}\right)^{k}$, the $k$-fold symmetric product of $\mathbf{T}_{4}$, containing divisors of the form

$$
D_{k}=\sum_{i=1}^{k}\left(t_{i}, s_{i}\right)
$$

and define the Abel map of such a divisor with base point $\infty$ :

$$
\mathbf{u}=\mathbf{u}\left(t_{1}, \ldots, t_{k}\right)=\sum_{i=1}^{k} \int_{\infty}^{t_{i}} \mathrm{~d} \mathbf{u} \bmod (\Lambda)
$$

If we set

$$
\Theta_{k}=\left\{\mathbf{u}: \mathbf{u}=\sum_{i=1}^{k} \int_{\infty}^{t_{i}} \mathrm{~d} \mathbf{u} \bmod (\Lambda)\right\}, \quad k \leq 4
$$

then evidently we have the stratification

$$
\operatorname{Jac}\left(\mathbf{T}_{4}\right)=\Theta_{4} \supset \Theta_{3} \supset \Theta_{2} \supset \Theta_{1} \supset \Theta_{0}=\mathbf{0} .
$$

We may let a point in $\Theta_{k}$ descend towards $\Theta_{k-1}$ by allowing $\left(t_{k}, s_{k}\right)$ to tend to $\infty$.

From the Jacobi inversion theorem, we know that one root of $\mathcal{P}$ must tend to infinity as $\mathbf{u}$ descends to $\Theta_{3}$, implying that $\sigma$ is zero there, so we can therefore define $\Theta_{3}$ equivalently by

$$
\Theta_{3}=\{\mathbf{u}: \sigma(\mathbf{u})=0\} .
$$

In principle this approach could be used to descend successively to lower strata, as was done in the hyperelliptic case, but this approach requires detailed knowledge of the partial differential equations satisfied by the $\wp_{i j}$. Instead, we use a theorem from a paper of Jorgenson [23] (but see also Fay [24], p.31 for a closely related result) to identify an alternative expression for $\Theta_{1}$ more directly.

This result is the following: Let

$$
\sum_{i=1}^{k} t_{k}
$$

be a divisor of degree $k<g$ on $\mathbf{C}_{g}$ and define its Abel map in the usual way:

$$
\mathbf{u}=\sum_{i=1}^{k} \int_{t_{a}}^{t_{k}} \mathrm{~d} \mathbf{u}
$$


Then the following equation holds:

$$
\frac{\sum_{j=1}^{g} \sigma_{j}(\mathbf{u}) a_{j}}{\sum_{j=1}^{g} \sigma_{j}(\mathbf{u}) b_{j}}=\frac{\operatorname{det}\left[\mathbf{a}\left|\mathrm{d} \mathbf{u}\left(t_{1}\right)\right| \cdots\left|\mathrm{d} \mathbf{u}\left(t_{k}\right)\right| \mathrm{d} \mathbf{u}(t)|\cdots| \mathrm{d} \mathbf{u}(t)^{(g-k-2)}\right]}{\operatorname{det}\left[\mathbf{b}\left|\mathrm{d} \mathbf{u}\left(t_{1}\right)\right| \cdots\left|\mathrm{d} \mathbf{u}\left(t_{k}\right)\right| \mathrm{d} \mathbf{u}(t)|\cdots| \mathrm{d} \mathbf{u}(t)^{(g-k-2)}\right]}
$$

where $\mathrm{d} \mathbf{u}(t)^{(i)}$ denotes the column of $i$-th derivatives of the holomorphic differentials $\mathrm{d} \mathbf{u}(t)$.

For the genus 4 trigonal curve $\mathbf{T}_{4}$ the set of holomorphic differentials is given by (24)

$$
\mathrm{d} \mathbf{u}^{\mathrm{T}}=\left(1, t, s, t^{2}\right) \frac{\mathrm{d} t}{3 s^{2}}
$$

and so we can construct the strata $\Theta_{k}$ successively as follows.

We have already noted that on $\Theta_{3}, \sigma(\mathbf{u})=0$. In that case (45) reduces to:

$$
\frac{\sum_{j=1}^{4} \sigma_{j}(\mathbf{u}) a_{j}}{\sum_{j=1}^{4} \sigma_{j}(\mathbf{u}) b_{j}}=\frac{\operatorname{det}\left[\mathbf{a}\left|\mathrm{d} \mathbf{u}\left(t_{1}\right)\right| \mathrm{d} \mathbf{u}\left(t_{2}\right) \mid \mathrm{d} \mathbf{u}\left(t_{3}\right)\right]}{\operatorname{det}\left[\mathbf{b}\left|\operatorname{d} \mathbf{u}\left(t_{1}\right)\right| \mathrm{d} \mathbf{u}\left(t_{2}\right) \mid \mathrm{d} \mathbf{u}\left(t_{3}\right)\right]} .
$$

Now as $\mathbf{u}$ in $\Theta_{3}$ approaches $\Theta_{2}$,

$$
t_{3} \rightarrow \infty \text {. }
$$

We can therefore express the fourth column of both determinants in terms of $\mathrm{d} u_{i}$ for $t_{3}$ near infinity. The local co-ordinate is $t_{3}=1 / \xi^{3}$ and so substituting this into (24) we find

$$
\begin{aligned}
\frac{\mathrm{d} u_{1}}{\mathrm{~d} \xi} & =-\xi^{6}+\frac{2}{3} \lambda_{4} \xi^{9}+O\left(\xi^{12}\right) \\
\frac{\mathrm{d} u_{2}}{\mathrm{~d} \xi} & =-\xi^{3}+\frac{2}{3} \lambda_{4} \xi^{6}+O\left(\xi^{9}\right) \\
\frac{\mathrm{d} u_{3}}{\mathrm{~d} \xi} & =-\xi+\frac{1}{3} \lambda_{4} \xi^{4}+O\left(\xi^{7}\right) \\
\frac{\mathrm{d} u_{4}}{\mathrm{~d} \xi} & =-1+\frac{2}{3} \lambda_{4} \xi^{3}+O\left(\xi^{6}\right) .
\end{aligned}
$$

Letting $\xi$ tend to zero, the determinant in the numerator of (46) becomes

$$
C\left|\begin{array}{cccc}
a_{1} & 1 & 1 & 0 \\
a_{2} & t_{1} & t_{2} & 0 \\
a_{3} & s_{1} & s_{2} & 0 \\
a_{4} & t_{1}^{2} & t_{2}^{2} & 1
\end{array}\right| .
$$

The denominator is of the same form but with $b_{i}$ instead of $a_{i}$. Evaluating the determinants gives

$$
\frac{\sum_{j=1}^{4} \sigma_{j}(\mathbf{u}) a_{j}}{\sum_{j=1}^{4} \sigma_{j}(\mathbf{u}) b_{j}}=\frac{a_{1}\left(t_{1} s_{2}-s_{1} t_{2}\right)+a_{2}\left(s_{1}-s_{2}\right)+a_{3}\left(t_{2}-t_{1}\right)}{b_{1}\left(t_{1} s_{2}-s_{1} t_{2}\right)+b_{2}\left(s_{1}-s_{2}\right)+b_{3}\left(t_{2}-t_{1}\right)} .
$$

This condition holds for $\mathbf{u}\left(t_{1}, t_{2}\right) \in \Theta_{2}$. Since $a_{4}$ and $b_{4}$ do not appear in the right hand side, we must set their coefficients to be zero and so the stratum $\Theta_{2}$ is characterised by

$$
\Theta_{2}=\left\{\mathbf{u}: \sigma(\mathbf{u})=\sigma_{4}(\mathbf{u})=0\right\} .
$$


In $\Theta_{2},(45)$ reads

$$
\frac{\sum_{j=1}^{4} \sigma_{j}(\mathbf{u}) a_{j}}{\sum_{j=1}^{4} \sigma_{j}(\mathbf{u}) b_{j}}=\frac{\operatorname{det}\left[\mathbf{a}\left|\mathrm{d} \mathbf{u}\left(t_{1}\right)\right| \mathrm{d} \mathbf{u}\left(t_{2}\right) \mid \mathrm{d} \mathbf{u}\left(t_{2}\right)^{1}\right]}{\operatorname{det}\left[\mathbf{b}\left|\operatorname{d} \mathbf{u}\left(t_{1}\right)\right| \operatorname{d} \mathbf{u}\left(t_{2}\right) \mid \mathrm{d} \mathbf{u}\left(t_{2}\right)^{(1)}\right]} .
$$

Now, as before, we let $t_{2} \rightarrow \infty$. The third column of the two determinants can be expanded, as before, in powers of $\xi=1 /\left(t_{2}\right)^{1 / 3}$. The fourth column is given by the derivatives of these expressions:

$$
\begin{aligned}
\frac{\mathrm{d}^{2} u_{1}}{\mathrm{~d} \xi^{2}} & =-6 \xi^{5}+6 \lambda_{4} \xi^{8}+O\left(\xi^{11}\right) \\
\frac{\mathrm{d}^{2} u_{2}}{\mathrm{~d} \xi^{2}} & =-3 \xi^{2}+4 \lambda_{4} \xi^{5}+O\left(\xi^{8}\right) \\
\frac{\mathrm{d}^{2} u_{3}}{\mathrm{~d} \xi^{2}} & =-1+\frac{4}{3} \lambda_{4} \xi^{3}+O\left(\xi^{6}\right) \\
\frac{\mathrm{d}^{2} u_{4}}{\mathrm{~d} \xi^{2}} & =-2 \lambda_{4} \xi^{2}+O\left(\xi^{5}\right) .
\end{aligned}
$$

Letting $\xi$ tend to zero, the numerator of (52) now becomes

$$
C\left|\begin{array}{cccc}
a_{1} & 1 & 0 & 0 \\
a_{2} & t_{1} & 0 & 0 \\
a_{3} & s_{1} & 0 & 1 \\
a_{4} & t_{1}^{2} & 1 & 0
\end{array}\right|
$$

and again the matrix in the denominator is of the same form but with $a_{i}$ replaced by $b_{i}$. Hence, equation (45) gives the relation

$$
\frac{\sum_{j=1}^{4} \sigma_{j}(\mathbf{u}) a_{j}}{\sum_{j=1}^{4} \sigma_{j}(\mathbf{u}) b_{j}}=\frac{a_{2}-a_{1} t_{1}}{b_{2}-b_{1} t_{1}}
$$

Since $a_{3}, a_{4}$ and $b_{3}, b_{4}$ do not appear in right hand side of this equation we must set their coefficients equal to zero.

It follows that the stratum $\Theta_{1}$ can therefore be characterised by

$$
\Theta_{1}=\left\{\mathbf{u}: \sigma(\mathbf{u})=\sigma_{4}(\mathbf{u})=\sigma_{3}(\mathbf{u})=0\right\}
$$

(see [25] for an analogous result for a $(2,5)$ curve), and we obtain the relation

$$
\frac{a_{1} \sigma_{1}(\mathbf{u})+a_{2} \sigma_{2}(\mathbf{u})}{b_{1} \sigma_{1}(\mathbf{u})+b_{2} \sigma_{2}(\mathbf{u})}=\frac{a_{2}-a_{1} t_{1}}{b_{2}-b_{1} t_{1}} .
$$

If we now set $a_{1}=1, a_{2}=0$ and $b_{1}=0, b_{2}=-1$, we find

$$
t_{1}=-\frac{\sigma_{1}}{\sigma_{2}}(\mathbf{u})
$$

which gives the inversion of the restriction of the Abel map to $\mathbf{T}_{4}$ :

$$
\mathbf{u}=\int_{\infty}^{t_{1}} \mathrm{~d} \mathbf{u}
$$

for $\mathbf{u} \in \Theta_{1}$, the one-dimensional stratum of the 4-dimensional Jacobian $\operatorname{Jac}\left(\mathbf{T}_{4}\right)$. 


\section{Evaluation of $\varphi$}

We can now transform integrand $(27) \varphi(t) \mathrm{d} t$ using the inversion formula (55)

$$
t=-\frac{\sigma_{1}}{\sigma_{2}}(\mathbf{u}) \quad \text { for } \quad \mathbf{u} \in \Theta_{1}
$$

and the expressions for the holomorphic differentials

$$
\mathrm{d} u_{1}=\frac{\mathrm{d} t}{3 s^{2}}, \quad \mathrm{~d} u_{2}=t \frac{\mathrm{d} t}{3 s^{2}}, \quad \mathrm{~d} u_{3}=s \frac{\mathrm{d} t}{3 s^{2}}, \quad \mathrm{~d} u_{4}=t^{2} \frac{\mathrm{d} t}{3 s^{2}} .
$$

From equation (27) we have

$$
\varphi(t) \mathrm{d} t=k\left(A_{2}+A_{3} t+A_{4} t^{2}\right) \frac{\mathrm{d} t}{3 s^{2}}+k\left(\frac{1}{t^{2}}+\frac{2}{3} \frac{\lambda_{1}}{\lambda_{0}} \frac{1}{t}\right) \frac{\mathrm{d} t}{3 s^{2}} .
$$

Separating this into the holomorphic and meromorphic parts $\varphi_{1}$ and $\varphi_{2}$, given respectively by

and

$$
\varphi_{1}=\left(A_{2}+A_{3} t+A_{4} t^{2}\right) \frac{\mathrm{d} t}{3 s^{2}}
$$

we see

$$
\varphi_{2}=\left(\frac{1}{t^{2}}+\frac{2}{3} \frac{\lambda_{1}}{\lambda_{0}} \frac{1}{t}\right) \frac{\mathrm{d} t}{3 s^{2}}
$$

$$
\varphi_{1}(t) \mathrm{d} t=k\left[A_{2} \mathrm{~d} u_{1}+A_{3} \mathrm{~d} u_{2}+A_{4} \mathrm{~d} u_{4}\right]
$$

and

$$
\varphi_{2}(t) \mathrm{d} t=k\left[\left(\frac{\sigma_{2}}{\sigma_{1}}(\mathbf{u})\right)^{2}-\frac{2}{3} \frac{\lambda_{1}}{\lambda_{0}} \frac{\sigma_{2}}{\sigma_{1}}(\mathbf{u})\right] \mathrm{d} u_{1} .
$$

Thus $\varphi_{1}$ is a sum of holomorphic Abelian differentials on the Riemann surface $\mathbf{T}_{4}$. Since $\varphi(p)$ has zero residue at $p=\infty$ on all three sheets, and residues are invariant under conformal maps, we know that the second term $\varphi_{2}$ must have a double pole with zero residue when $\sigma_{1}(\mathbf{u})=0$; modulo periods; there are three such points, denoted $\mathbf{u}_{\mathbf{0}}, \omega \mathbf{u}_{\mathbf{0}}$, and $\omega^{2} \mathbf{u}_{\mathbf{0}}$, where $\omega=-\frac{1}{2}+i \frac{\sqrt{3}}{2}$, corresponding to one point on each sheet of $\mathbf{T}_{4} \cdot \varphi_{2}$ is regular everywhere else on $\Theta_{1}$.

Thus as in the hyperelliptic cases we must construct a function $\Psi$ which satisfies

$$
\frac{\mathrm{d}}{\mathrm{d} u_{1}}[\Psi(\mathbf{u})]=\varphi_{2}(\mathbf{u}), \quad \mathbf{u} \in \Theta_{1} .
$$

As the integral of a second kind differential, it can have at worst simple poles at the three points $\omega^{i} \mathbf{u}_{\mathbf{0}}$. From the holomorphic differentials (56), we have

$$
\frac{\partial}{\partial u_{2}}=t \frac{\partial}{\partial u_{1}}, \quad \frac{\partial}{\partial u_{3}}=s \frac{\partial}{\partial u_{1}}, \quad \frac{\partial}{\partial u_{4}}=t^{2} \frac{\partial}{\partial u_{1}}
$$

and so the differential operator $D_{1}=\mathrm{d} /\left.\mathrm{d} u_{1}\right|_{\Theta_{1}}$ is given by

$$
\begin{aligned}
D_{1} & =\left.\frac{\mathrm{d}}{\mathrm{d} u_{1}}\right|_{\Theta_{1}} \\
& =\frac{\partial}{\partial u_{1}}+t \frac{\partial}{\partial u_{2}}+s \frac{\partial}{\partial u_{3}}+t^{2} \frac{\partial}{\partial u_{4}} \\
& =\frac{\partial}{\partial u_{1}}-\left(\frac{\sigma_{1}}{\sigma_{2}}\right) \frac{\partial}{\partial u_{2}}+s \frac{\partial}{\partial u_{3}}+\left(\frac{\sigma_{1}}{\sigma_{2}}\right)^{2} \frac{\partial}{\partial u_{4}}
\end{aligned}
$$


where

$$
\begin{aligned}
s^{3} & =\left[t^{5}+\lambda_{4} t^{4}+\lambda_{3} t^{4}+\lambda_{2} t^{2}+\lambda_{1} t+\lambda_{0}\right] \\
& =\left[-\left(\frac{\sigma_{1}}{\sigma_{2}}\right)^{5}+\lambda_{4}\left(\frac{\sigma_{1}}{\sigma_{2}}\right)^{4}-\lambda_{3}\left(\frac{\sigma_{1}}{\sigma_{2}}\right)^{3}+\lambda_{2}\left(\frac{\sigma_{1}}{\sigma_{2}}\right)^{2}-\lambda_{1}\left(\frac{\sigma_{1}}{\sigma_{2}}\right)+\lambda_{0}\right] .
\end{aligned}
$$

Since this has 3 distinct roots for $s$, we cannot compare $D_{1}(\Psi)$ and $\varphi_{2}$ directly. This problem is therefore approached in a similar way to the higher genus hyperelliptic cases. We begin by identifying a function $\Psi$ whose derivative has the same expansion as $\varphi_{2}$ near each of the poles $\omega^{i} \mathbf{u}_{0}$. We then verify that this function has no other poles. The solution can then be obtained by using an extension of Liouville's theorem on the stratum $\Theta_{1}$ of the Jacobi variety.

\subsection{Expansion near the pole $\mathbf{u}_{0}$.}

We will begin by expanding the function $\varphi_{2}$ near the point $\mathbf{u}=\mathbf{u}_{0}$ and then compare this with the expansion of a suitable function $\Psi$. We note that because of the cyclic automorphism of $\mathbf{T}_{4}$, the expansions at the other 2 points $\omega^{n} \mathbf{u}_{0}$ are not essentially different; the conditions to be imposed at the three points $\omega^{n} \mathbf{u}_{0}$ all hold or fail together.

Let $\mathbf{u}_{0}=\left(u_{0,1}, u_{0,2}, u_{0,3}, u_{0,4}\right)$, then the Taylor series of the terms in $\varphi_{2}$ are given as follows. Writing $w_{i}=\left(u_{i}-u_{0, i}\right)$ we have

$$
\begin{aligned}
&-\frac{2}{3} \frac{\lambda_{1}}{\lambda_{0}} \frac{\sigma_{2}}{\sigma_{1}}=-\frac{2}{3} \frac{\lambda_{1}}{\lambda_{0}}\left[\frac{\sigma_{2}+\sigma_{12} w_{1}+\sigma_{23} w_{3}+1 / 2 \sigma_{112} w_{1}^{2}+\ldots}{\sigma_{11} w_{1}+\sigma_{13} w_{3}+1 / 2 \sigma_{111} w_{1}^{2}+\sigma_{113} w_{1} w_{3}+\sigma_{12} w_{2}+1 / 2 \sigma_{133} w_{3}^{2} \ldots}\right], \\
&\left(\frac{\sigma_{2}}{\sigma_{1}}\right)^{2}=\left[\frac{\sigma_{2}^{2}+2 \sigma_{2} \sigma_{12} w_{1}+2 \sigma_{2} \sigma_{23} w_{3}+\ldots}{\sigma_{11} w_{1}^{2}+2 \sigma_{13} \sigma_{11} w_{1} w_{3}+\sigma_{13}^{2} w_{3}^{2}+\ldots}\right] .
\end{aligned}
$$

Since this expansion is on $\Theta_{1}$, we can rewrite it in terms of the single parameter $t$. On $\Theta_{1}$ we know $t=-\sigma_{1} / \sigma_{2}$ and so $\sigma_{1}\left(\mathbf{u}_{0}\right)=0$ corresponds to the points $t=0$. This means that the $u_{0, i}$ are given by the integrals

$$
u_{0, i}=\int_{\infty}^{0} \mathrm{~d} u_{i} \quad i=1, \ldots, 4
$$

As the Riemann surface $\mathbf{T}_{4}$ has no singularities and the branch points satisfy $T_{i}>0$, $t=0$ is a regular point and so we write the $w_{i}=u_{i}-\left(u_{0}\right)_{i}$ in terms of the local parameter $t$. This gives, on the sheet on which $s \rightarrow \lambda_{0}^{1 / 3}$ as $t \rightarrow 0$,

$$
\begin{aligned}
w_{1} & =\int_{\infty}^{t} \mathrm{~d} u_{1}-\int_{\infty}^{0} \mathrm{~d} u_{1}=\int_{0}^{t} \mathrm{~d} u_{1} \\
& =\int_{0}^{t} \frac{\mathrm{d} t^{\prime}}{\left(s^{\prime}\right)^{2 / 3}} \\
& =\frac{1}{3 \lambda_{0}^{2 / 3}} t-\frac{1}{9} \frac{\lambda_{1}}{\lambda_{0}^{5 / 3}} t^{2}+\mathrm{O}\left(t^{3}\right),
\end{aligned}
$$

with similar formulae on the other 2 sheets. Similarly, we see that

$$
w_{2}=\frac{1}{6} \frac{1}{\lambda_{0}^{(2 / 3)}} t^{2}+\mathrm{O}\left(t^{3}\right),
$$




$$
w_{3}=\frac{1}{3} \frac{1}{\lambda_{0}^{(1 / 3)}} t+\mathrm{O}\left(t^{2}\right)
$$

and

$$
w_{4}=\frac{1}{9} \frac{1}{\lambda_{0}^{(2 / 3)}} t^{3}+\mathrm{O}\left(t^{4}\right) .
$$

We can thus rewrite the series (60) - (62) in terms of the parameter $w_{1}$. This gives

$$
\begin{aligned}
& w_{2}=\left(u_{2}-u_{0,2}\right)=\frac{3}{2} \lambda_{0}^{(2 / 3)} w_{1}^{2}+\mathrm{O}\left(w_{1}^{3}\right), \\
& w_{3}=\left(u_{3}-u_{0,3}\right)=\lambda_{0}^{(1 / 3)} w_{1}+\mathrm{O}\left(w_{1}^{2}\right), \\
& w_{4}=\left(u_{4}-u_{0,4}\right)=3 \lambda_{0}^{(4 / 3)} w_{1}^{3}+\mathrm{O}\left(w_{1}^{4}\right) .
\end{aligned}
$$

Substituting in the expressions for $w_{i}$ in terms of $w_{1}$ (63)-(65) we obtain the following expansions:

$$
\begin{aligned}
& -\frac{2}{3} \frac{\lambda_{1}}{\lambda_{0}} \frac{\sigma_{2}}{\sigma_{1}}=-\frac{2}{3} \frac{\lambda_{1}}{\lambda_{0}}\left[\left(\frac{\sigma_{2}}{\sigma_{11}+\lambda_{0}^{1 / 3} \sigma_{13}}\right) \frac{1}{w_{1}}+\mathrm{O}(1)\right] \\
& \left(\frac{\sigma_{2}}{\sigma_{1}}\right)^{2}=\left[\left(\frac{\sigma_{2}^{2}}{\left(\sigma_{11}+\lambda_{0}^{1 / 3} \sigma_{13}\right)^{2}}\right) \frac{1}{w_{1}^{2}}+\mathbf{C} \frac{1}{w_{1}}+\mathrm{O}(1)\right]
\end{aligned}
$$

where the coefficient $\mathbf{C}$ is given by

$$
\begin{aligned}
& \mathbf{C}=\frac{-\sigma_{2}}{\left[\sigma_{11}+\left(\lambda_{0}^{1 / 3}\right) \sigma_{13}\right]^{4}} \times \\
& {\left[-2 \sigma_{12} \sigma_{11}^{2}-2\left(\lambda_{0}^{2 / 3}\right) \sigma_{12} \sigma_{13}^{2}\right.} \\
& -4\left(\lambda_{0}^{1 / 3}\right) \sigma_{12} \sigma_{13} \sigma_{11}-2\left(\lambda_{0}^{1 / 3}\right) \sigma_{23} \sigma_{11}^{2} \\
& -2 \lambda_{0} \sigma_{23} \sigma_{13}^{2}-4\left(\lambda_{0}^{2 / 3}\right) \sigma_{23} \sigma_{13} \sigma_{11} \\
& +\sigma_{2} \sigma_{11} \sigma_{111}+\left(\lambda_{0}^{2 / 3}\right) \sigma_{2} \sigma_{11} \sigma_{133} \\
& +2\left(\lambda_{0}^{1 / 3}\right) \sigma_{2} \sigma_{11} \sigma_{113}+2\left(\lambda_{0}^{2 / 3}\right) \sigma_{2} \sigma_{13} \sigma_{113} \\
& +\lambda_{1} \sigma_{2} \sigma_{13} \sigma_{11}+\lambda_{0} \sigma_{2} \sigma_{13} \sigma_{133} \\
& +3\left(\lambda_{0}^{2 / 3}\right) \sigma_{2} \sigma_{11} \sigma_{12}+\left(\lambda_{1} \lambda_{0}^{2 / 3}\right) \sigma_{2} \sigma_{13}^{2} \\
& \left.+3 \sigma_{2} \sigma_{13} \sigma_{12}\right] .
\end{aligned}
$$

The second and third order sigma derivatives in this term are

$$
\sigma_{11}, \quad \sigma_{12}, \quad \sigma_{13}, \quad \sigma_{23}
$$

and

$$
\sigma_{111}, \quad \sigma_{113}, \quad \sigma_{133}
$$

Thus to check that our integrand $\varphi_{2}$ has zero residue, as it must, we need to find lower order expressions for these derivatives at the point $\mathbf{u}_{0} \in \Theta_{1}$. First we will find some relations holding throughout $\Theta_{1}$, and then specialise to the 3 points $\omega^{n} \mathbf{u}_{0}$. 
4.1.1. Relations between the $\sigma$-derivatives holding throughout $\Theta_{1}$. We start in $\Theta_{3}$. The point at infinity is a branch point of period 3 and so the expansion for $t_{3}$ is given in terms of the local parameter $t_{3}=1 / \xi^{3}$. Substituting this into the definitions of $u_{i}$ from the Abel map,

$$
u_{i}\left(t_{1}, t_{2}, 1 / \xi^{3}\right)-u_{i}\left(t_{1}, t_{2}, \infty\right)=\int_{\infty}^{1 / \xi^{3}} \mathrm{~d} u_{i}
$$

we find

$$
\begin{aligned}
& u_{1}\left(t_{1}, t_{2}, 1 / \xi^{3}\right)-u_{1}\left(t_{1}, t_{2}\right)=-\frac{1}{7} \xi^{7}+\frac{1}{15} \lambda_{4} \xi^{10}+\mathrm{O}\left(\xi^{13}\right) \\
& u_{2}\left(t_{1}, t_{2}, 1 / \xi^{3}\right)-u_{2}\left(t_{1}, t_{2}\right)=-\frac{1}{4} \xi^{4}+\frac{2}{21} \lambda_{4} \xi^{7}+\mathrm{O}\left(\xi^{10}\right) \\
& u_{3}\left(t_{1}, t_{2}, 1 / \xi^{3}\right)-u_{3}\left(t_{1}, t_{2}\right)=-\frac{1}{2} \xi^{2}+\frac{1}{15} \lambda_{4} \xi^{5}+\mathrm{O}\left(\xi^{8}\right)
\end{aligned}
$$

and

$$
u_{4}\left(t_{1}, t_{2}, 1 / \xi^{3}\right)-u_{4}\left(t_{1}, t_{2}\right)=-\xi+\frac{1}{6} \lambda_{4} \xi^{4}+\mathrm{O}\left(\xi^{7}\right)
$$

If we now calculate the Taylor series for $\sigma(\mathbf{u})=0$, which holds identically in $\Theta_{3}$, in terms of $\xi$ using (70), and then substitute in identities (67) - (70) we obtain

$$
\begin{aligned}
0= & \sigma\left(u\left(t_{1}, t_{2}, \infty\right)-\left[u\left(t_{1}, t_{2}, \infty\right)-u\left(t_{1}, t_{2}, t_{3}\right)\right]\right) \\
\simeq & \sigma\left(u\left(t_{1}, t_{2}, \infty\right)\right)+\left(-\sigma_{4}\left(u\left(t_{1}, t_{2}, \infty\right)\right)\right) \xi \\
& +\left(-\frac{1}{2} \sigma_{3}\left(u\left(t_{1}, t_{2}, \infty\right)\right)+\frac{1}{2} \sigma_{44}\left(u\left(t_{1}, t_{2}, \infty\right)\right)\right) \xi^{2}+\mathrm{O}\left(\xi^{3}\right) \\
= & \left(-\frac{1}{2} \sigma_{3}\left(u\left(t_{1}, t_{2}, \infty\right)\right)+\frac{1}{2} \sigma_{44}\left(u\left(t_{1}, t_{2}, \infty\right)\right)\right) \xi^{2}+\mathrm{O}\left(\xi^{3}\right)
\end{aligned}
$$

since $\sigma_{4}=\sigma=0$ on $\Theta_{2}$.

The terms in the right hand side are evaluated at the point $\mathbf{u}=\mathbf{u}\left(t_{1}, t_{2}, \infty\right) \in \Theta_{2}$. Setting the coefficients of $\xi$ equal to zero, we find

$$
\sigma_{44}-\sigma_{3}=0, \quad \forall \mathbf{u} \in \Theta_{2} .
$$

on $\Theta_{2}$. If we repeat this process, expanding (74) as $\mathbf{u} \rightarrow \Theta_{1} \Leftrightarrow t_{2} \rightarrow \infty$, we similarly obtain the relation

$$
\sigma_{33}-\sigma_{2}=0, \quad \forall \mathbf{u} \in \Theta_{1} .
$$

4.1.2. Relations between the $\sigma$-derivatives holding at $\mathbf{u}_{0} \in \Theta_{1}$. At the point $\mathbf{u}=\mathbf{u}_{0}$ we have the additional restriction $\sigma_{1}\left(\mathbf{u}_{0}\right)=0$. This will yield enough relations to evaluate the expansion of $\varphi_{2}$.

We have

$$
\Theta_{1}=\left\{\mathbf{u}: \sigma(\mathbf{u})=\sigma_{4}(\mathbf{u})=\sigma_{3}(\mathbf{u})=0\right\}
$$

and the terms we need to express in terms of lower derivatives are

$$
\sigma_{11}, \quad \sigma_{12}, \quad \sigma_{13}, \quad \sigma_{23},
$$


and

$$
\sigma_{111}, \quad \sigma_{113}, \quad \sigma_{133}
$$

If we were to expand $\sigma_{4}(\mathbf{u})=0 \quad \forall \mathbf{u} \in \Theta_{1}$ for $\mathbf{u}$ near $\mathbf{u}_{0}$, then all of its terms would contain derivatives with respect to $u_{4}$, which we do not require. Therefore we will just consider the identities $\sigma=0$ and $\sigma_{3}=0$, both valid throughout $\Theta_{1}$, and in particular near $\mathbf{u}_{0}$.

The stratum $\Theta_{3}$ is given by the set of points $\mathbf{u} \in \operatorname{Jac}\left(\mathbf{T}_{4}\right)$ such that

$$
\mathbf{u}\left(t_{1}, t_{2}, t_{3}\right)=\sum_{i=1}^{3} \int_{\infty}^{t_{i}} \mathrm{~d} \mathbf{u}
$$

where the divisor $\left(t_{1}+t_{2}+t_{3}-3 \infty\right)$ has dimension 3 . This can also be defined by condition (44):

$$
\Theta_{3}=\{\mathbf{u}: \sigma(\mathbf{u})=0\} .
$$

We begin by calculating the Taylor series of

$$
\sigma(\mathbf{u})=0
$$

for $\mathbf{u} \in \Theta_{1}$ near the point $\mathbf{u}_{0}$. This gives

$$
\begin{aligned}
0 & =\sigma\left(\mathbf{u}_{0}+\left(\mathbf{u}-\mathbf{u}_{0}\right)\right) \\
& =\sigma+\left(\sigma_{1}\right) w_{1}+\left(\sigma_{3}\right) w_{3}+\left(\frac{1}{2} \sigma_{11}\right) w_{1}^{2} \\
& +\left(\sigma_{13}\right) w_{1} w_{3}+\left(\sigma_{2}\right) w_{2}+\left(\frac{1}{2} \sigma_{33}\right) w_{3}^{2}+\cdots
\end{aligned}
$$

where $w_{i}=\left(u_{i}-u_{0, i}\right)(i=1, \ldots, 4)$. If we now substitute in the expressions for $w_{i}$ as functions of $w_{1}(60)-(62)$ and use the results valid at $\mathbf{u}_{0} \in \Theta_{1}$ :

$$
\sigma_{1}\left(\mathbf{u}_{0}\right)=\sigma_{3}\left(\mathbf{u}_{0}\right)=\sigma_{4}\left(\mathbf{u}_{0}\right)=\sigma\left(\mathbf{u}_{0}\right)=0,
$$

we find

$$
\begin{aligned}
0= & \sigma\left[\sigma_{3} \lambda_{0}^{(1 / 3)}+\sigma_{1}\right] w_{1} \\
& +\left[\sigma_{13} \lambda_{0}^{(1 / 3)}+\frac{1}{2} \sigma_{3} \lambda_{3}+\frac{1}{2} \lambda_{0}^{(2 / 3)} \sigma_{33}+\frac{1}{2} \sigma_{11}+\frac{3}{2} \sigma_{2} \lambda_{0}^{(2 / 3)}\right] w_{1}^{2}+\mathrm{O}\left(w_{1}^{3}\right) \\
= & {\left[\frac{3}{2} \sigma_{2} \lambda_{0}^{(2 / 3)}+\frac{1}{2} \sigma_{11}+\sigma_{13} \lambda_{0}^{(1 / 3)}+\frac{1}{2} \lambda_{0}^{(2 / 3)} \sigma_{33}\right] w_{1}^{2}+\mathrm{O}\left(w_{1}^{3}\right) }
\end{aligned}
$$

where each term on the right hand side is evaluated at $\mathbf{u}_{0}$. Setting the leading coefficient, that of $w_{1}^{2}$, in (76) to be zero gives

$$
\sigma_{11}=-3 \lambda_{0}^{(2 / 3)} \sigma_{2}-2 \lambda_{0}^{(1 / 3)} \sigma_{13}-\lambda_{0}^{(2 / 3)} \sigma_{33}
$$

at $\mathbf{u}_{0} \in \Theta_{1}$. An analogous relation for $\sigma_{111}$ can be found from the coefficient of $w_{1}^{3}$.

The substitutions for $\sigma_{13}$ and $\sigma_{113}$ are then obtained from the Taylor series of $\sigma_{3}(\mathbf{u})=0$ for $\mathbf{u} \in \Theta_{1}$ near $\mathbf{u}_{0}$. 
Now these still involve $\sigma_{33}$ and $\sigma_{133}$. The former was found above (75), which holds throughout $\Theta_{1}$. Expanding near $\mathbf{u}=\mathbf{u}_{0}$ gives the required result.

To summarise, the full list of substitutions required to evaluate $\mathbf{C}$ is

$$
\begin{aligned}
\sigma_{11} & =-2 \lambda_{0}^{(2 / 3)} \sigma_{2}, \\
\sigma_{13} & =-\lambda_{0}^{(1 / 3)} \sigma_{2}, \\
\sigma_{33} & =\sigma_{2}, \\
\sigma_{111} & =-6 \lambda_{0}^{(2 / 3)} \sigma_{12}-3 \lambda_{0}^{(1 / 3)} \lambda_{3} \sigma_{2}, \\
\sigma_{113} & =-2 \lambda_{0}^{(2 / 3)} \sigma_{23}-2 \lambda_{0}^{(1 / 3)} \sigma_{12}-\lambda_{3} \sigma_{2}, \\
\sigma_{133} & =-2 \lambda_{0}^{(1 / 3)} \sigma_{23}+\sigma_{12} .
\end{aligned}
$$

valid for $\mathbf{u}=\mathbf{u}_{0} \in \Theta_{1}$.

If we substitute these into the coefficient $\mathbf{C}(66)$, then this term vanishes. Thus the expression for $\varphi \mathrm{dt}$ is indeed a second kind differential on $\Theta_{1}$ :

$$
\varphi\left(\mathbf{u}_{0}-\left(\mathbf{u}_{0}-\mathbf{u}\right)\right)=\left(\frac{1}{9} \frac{1}{\lambda_{0}^{4 / 3}}\right) \frac{1}{w_{1}^{2}}+O\left(w_{1}^{0}\right) .
$$

We now consider the function

$$
\Psi(\mathbf{u})=\frac{\sigma_{13}}{\sigma_{1}}(\mathbf{u})
$$

Let

$$
\psi(\mathbf{u})=\frac{\mathrm{d}}{\mathrm{d} u_{1}}[\Psi(\mathbf{u})]=\left(\frac{\partial}{\partial u_{1}}+t \frac{\partial}{\partial u_{2}}+s \frac{\partial}{\partial u_{3}}+t^{2} \frac{\partial}{\partial u_{4}}\right) \Psi(\mathbf{u})
$$

where

$$
s^{3}=\left(t^{5}+\lambda_{4} t^{4}+\lambda_{3} t^{3}+\lambda_{2} t^{2}+\lambda_{1} t+\lambda_{0}\right) .
$$

Substituting

$$
t=-\frac{\sigma_{1}}{\sigma_{2}}(\mathbf{u})
$$

into $\psi(\mathbf{u})$ we see

$\psi=-\frac{1}{\sigma_{1}^{2}}\left(s \sigma_{13}^{2}+\sigma_{13} \sigma_{11}\right)+\frac{1}{\sigma_{1}}\left(\sigma_{113}+s \sigma_{133}+\frac{\sigma_{13} \sigma_{12}}{\sigma_{2}}\right)-\frac{\sigma_{123}}{\sigma_{2}}+\frac{\sigma_{1} \sigma_{134}-\sigma_{13} \sigma_{14}}{\sigma_{2}^{2}}$.

Since $s$ is regular at $\mathbf{u}=\mathbf{u}_{0}$, all singularities must come from the coefficients of $\sigma_{1}^{-2}$ and $\sigma_{1}^{-1}$. Thus for $\mathbf{u}$ near $\mathbf{u}_{0}$ we will write $s_{0}=\left.s(t)\right|_{t \simeq 0}$ and define

$$
\psi_{0}(\mathbf{u})=\left[-\frac{1}{\sigma_{1}^{2}}\left(s_{0} \sigma_{13}^{2}+\sigma_{13} \sigma_{11}\right)+\frac{1}{\sigma_{1}}\left(\sigma_{113}+s_{0} \sigma_{133}+\frac{\sigma_{13} \sigma_{12}}{\sigma_{2}}\right)\right]+O(1) .
$$

To expand this near $\mathbf{u}=\mathbf{u}_{0}$ we first need to evaluate $s_{0}$. Using Maple, we calculate the Taylor series of $s_{0}$ for $t$ near zero. The first few terms are

$$
s_{0}=\lambda_{0}^{1 / 3}+\left(\frac{1}{3} \frac{\lambda_{1}}{\lambda_{0}^{2 / 3}}\right) t+\left(\frac{1}{3} \frac{\lambda_{2}}{\lambda_{0}^{2 / 3}}-\frac{1}{9} \frac{\lambda_{1}^{2}}{\lambda_{0}^{5 / 3}}\right) t^{2}+O\left(t^{3}\right) .
$$


We can then invert the series for $w_{1}(t)(59)$ to rewrite this as

$$
s_{0}=\lambda_{0}^{1 / 3}+\left(\lambda_{3}\right) w_{1}+\left(3 \lambda_{0}^{2 / 3} \lambda_{2}\right) w_{1}^{2}+O\left(w_{1}^{3}\right) .
$$

Using expression (87) for $s_{0}$, we can now expand $\psi_{0}$ for $\mathbf{u}$ near $\mathbf{u}_{0}$ in the same manner as $\varphi$. We obtain

$$
\psi_{0}=\left[-\sigma_{13}\left(\frac{\sigma_{11} \lambda_{0}^{2 / 3}+\lambda_{0} \sigma_{13}}{2 \sigma_{13} \sigma_{11} \lambda_{0}+\sigma_{13}^{2} \lambda_{0}^{4 / 3}+\sigma_{11}^{2} \lambda_{0}^{2 / 3}}\right)\right]\left(\frac{1}{w_{1}^{2}}\right)+O\left(w_{1}^{0}\right) .
$$

This can be simplified by writing $\sigma_{11}$ and $\sigma_{13}$ in terms of $\sigma_{2}$. From identities (77) and (78), we see

$$
\psi_{0}=\left[-\frac{1}{3} \frac{1}{\lambda_{0}^{1 / 3}}\right]\left(\frac{1}{w_{1}^{2}}\right)+O\left(w_{1}^{0}\right)
$$

and so the function

$$
-\frac{1}{3} \frac{1}{\lambda_{0}} \psi(\mathbf{u})=\frac{\mathrm{d}}{\mathrm{d} u_{1}}\left[-\frac{1}{3} \frac{1}{\lambda_{0}} \frac{\sigma_{13}}{\sigma_{1}}(\mathbf{u})\right]
$$

has same principal part as $\varphi_{2}$ near $\mathbf{u}=\mathbf{u}_{0}$ (see equation (83)).

\section{The expansion of $\sigma(\mathbf{u})$ near $\mathbf{u}=0$}

We now need to verify that the function

$$
\frac{\mathrm{d}}{\mathrm{d} u_{1}}\left[-\frac{1}{3} \frac{1}{\lambda_{0}} \frac{\sigma_{13}}{\sigma_{1}}(\mathbf{u})\right]
$$

like $\varphi_{2}$, is regular at $\mathbf{u}=\mathbf{0}$.

To do this we need the expansion of $\sigma$ for $\mathbf{u}$ near $\mathbf{0}$. Such an expansion for a $\sigma$ function was first found in the elliptic case by Weierstrass [26], [27]. A similar expansion was found for the genus 2 hyperelliptic case by Baker [28], and was recently generalised to arbitrary genus hyperelliptic curves by Buchstaber and Leykin [29].

In this case the leading terms of $\sigma$ can be evaluated as follows. We know that the first term in the Taylor expansion of $\sigma$ is the Schur-Weierstrass polynomial. To calculate this polynomial we proceed as follows. For a general point on $\operatorname{Jac}\left(\mathbf{T}_{4}\right)$ we have

$$
u_{i}\left(t_{1}, t_{2}, t_{3}, t_{4}\right)=\sum_{k=1}^{4} u_{i}\left(t_{k}\right) \quad i=1, \ldots, 4 .
$$

Let us now study the stratum $\Theta_{3}$ near $\mathbf{u}=\mathbf{0}$. We let $t_{1}, t_{2}$, and $t_{3}$ approach infinity, and set $t_{4}=\infty$. We replace $u_{i}\left(t_{1}, t_{2}, t_{3}, \infty\right)$, by the leading term in the expansion of the Abel map:

$$
\begin{aligned}
& u_{1}\left(t_{1}, t_{2}, t_{3}, \infty\right)=\left(-\frac{1}{7} \xi_{1}^{7}-\frac{1}{7} \xi_{2}^{7}-\frac{1}{7} \xi_{3}^{7}\right) \\
& u_{2}\left(t_{1}, t_{2}, t_{3}, \infty\right)=\left(-\frac{1}{4} \xi_{1}^{4}-\frac{1}{4} \xi_{2}^{4}-\frac{1}{4} \xi_{3}^{4}\right) \\
& u_{3}\left(t_{1}, t_{2}, t_{3}, \infty\right)=\left(-\frac{1}{2} \xi_{1}^{2}-\frac{1}{2} \xi_{2}^{2}-\frac{1}{2} \xi_{3}^{2}\right) \\
& u_{4}\left(t_{1}, t_{2}, t_{3}, \infty\right)=\left(-\xi_{1}-\xi_{2}-\xi_{3}\right) .
\end{aligned}
$$


If we evaluate the resultants of these equations, successively with respect to $\xi_{3}, \xi_{2}$ and then $\xi_{1}$, we obtain the polynomial

$$
S=C u_{4}^{12}\left(448 u_{2}^{2}-56 u_{3}^{2} u_{4}^{4}-112 u_{3}^{4}+448 u_{2} u_{3} u_{4}^{2}+u_{4}^{8}-448 u_{4} u_{1}\right)
$$

where $C \in \mathbb{R}$ is an irrelevant constant. The Schur-Weierstrass polynomial is the leading term in $\sigma$, which must vanish on $\Theta_{3}$. Now the factor $u_{4}^{12}$ is non-vanishing except at the origin. The Schur-Weierstrass polynomial for $\mathbf{T}_{4}$ is thus given by the factor

$$
S W=448 u_{2}^{2}-56 u_{3}^{2} u_{4}^{4}-112 u_{3}^{4}+448 u_{2} u_{3} u_{4}^{2}+u_{4}^{8}-448 u_{4} u_{1} .
$$

Now the weights of the terms $u_{i}$ are given by the Weierstrass gap sequence. These are 7, 4, 2 and 1 respectively and so this polynomial has weight 8 .

We now use this as the starting point for the Taylor series expansion of $\sigma$ near $\mathbf{u}=\mathbf{0}$. From (67)-(70) we see that the powers of $\xi$ in the Taylor series expansion of $u_{i}$ near $\mathbf{u}=\mathbf{0}$ increase by steps of 3 . Thus the total weights of the successive terms in the Taylor series for $\sigma$ near $\mathbf{u}=\mathbf{0}$ will increase by weights of 3 .

We look for a series for $\sigma$ as a sum of monomials of weights $8+3 n$ with $n \geq 0$, with coefficients of $u_{1}^{n^{1}} u_{2}^{n_{2}} u_{3}^{n_{3}} u_{4}^{n^{4}}$ which are isobaric polynomials in the $\lambda_{i}$ - they have weight of $\lambda_{i}$ is $3 i-15$. To include all the information about the curve, this series needs to contain all of the curve moduli $\lambda_{i}$. The lowest weight at which all $\lambda_{i}$ appear is 23, so we must calculate at least to this order.

There is a unique series, $\tilde{\sigma}$, including terms of weight $\leq 23$, which satisfies the conditions:

- The leading term, of $\mathrm{O}\left(u_{4}^{8}\right)$ of $\tilde{\sigma}$, is the Schur-weierstrass polynomial.

- $\tilde{\sigma}=\mathrm{O}\left(u_{4}^{26}\right)$ on $\Theta_{1}, \Theta_{2}$, and $\Theta_{3}$.

- On Jac $\left(\mathbf{T}_{4}\right)$, the Jacobi inversion formulae are satisfied to sufficiently high order:

$$
\wp_{14}+\wp_{24} t_{1}+\wp_{34} s_{1}+\wp_{44} t_{1}^{2}-t_{1} s_{1}=0
$$

and

$$
\left(\wp_{13}+\wp_{144}\right)+\left(\wp_{23}+\wp_{244}\right) t_{1}+\left(\wp_{33}+\wp_{344}\right) s_{1}+\left(\wp_{43}+\wp_{444}\right) t_{1}^{2}=t_{1}^{3} .
$$

Here the Kleinian $\wp$-functions are to be replaced by the corresponding logarithmic derivatives of $\tilde{\sigma}$, and evaluated at $\mathbf{u}\left(\left(t_{1}, s_{1}\right),\left(t_{2}, s_{2}\right),\left(t_{3}, s_{3}\right),\left(t_{4}, s_{4}\right)\right)$, where all points $t_{i}$, are allowed to tend to $\infty$, so that $\mathbf{u} \rightarrow \mathbf{0}$.

This series is given in full in the appendix.

Using this Taylor series expansion for $\sigma(\mathbf{u})$ as $\mathbf{u} \rightarrow \mathbf{0}$ we can quickly compare the properties of

$$
\varphi_{2}(\mathbf{u})=\left(\frac{\sigma_{2}}{\sigma_{1}}\right)^{2}-\frac{2}{3} \frac{\lambda_{3}}{\lambda_{0}} \frac{\sigma_{2}}{\sigma_{1}}
$$

and

$$
\frac{\mathrm{d}}{\mathrm{d} u_{1}} \Psi(\mathbf{u})=\frac{\mathrm{d}}{\mathrm{d} u_{1}} \frac{\sigma_{13}}{\sigma_{1}}
$$


for $\mathbf{u}$ near $\mathbf{0 .}$

We represent the sigma derivatives in terms of the known Taylor series $\tilde{\sigma}$ valid near $\mathbf{u}=\mathbf{0}$. The derivatives of $\sigma$ are determined up to order:

$$
\sigma_{i}=\tilde{\sigma}_{i}+\mathrm{O}\left(u_{4}^{26-\gamma_{i}}\right) .
$$

The leading terms are of order

$$
(S W)_{i}=\mathrm{O}\left(u_{4}^{8-\gamma_{i}}\right)
$$

Since $u_{1}$ has weight

$$
W\left(u_{1}\right)=\gamma_{1}=7,
$$

it follows that the series for $\varphi(\mathbf{u})$ is valid up to and including terms of weight 16 . It is given by

$$
\lim _{\mathbf{u} \rightarrow \mathbf{0}} \varphi(\mathbf{u})=\lim _{\xi \rightarrow 0}\left(\frac{2}{3} \frac{\lambda_{1}}{\lambda_{0}} \xi^{3}+\xi^{6}+\mathrm{O}\left(\xi^{17}\right)\right) .
$$

We can calculate the expansion for

$$
\begin{aligned}
D_{1}(\Psi(\mathbf{u}))= & D_{1}\left(\frac{\sigma_{13}}{\sigma_{1}}\right) \\
= & \left(\frac{\partial}{\partial u_{1}}-\frac{\sigma_{1}}{\sigma_{2}} \frac{\partial}{\partial u_{2}}+s \frac{\partial}{\partial u_{3}}+\left(\frac{\sigma_{1}}{\sigma_{2}}\right)^{2} \frac{\partial}{\partial u_{4}}\right) \frac{\sigma_{13}}{\sigma_{1}} \\
= & \frac{\sigma_{113}}{\sigma_{1}}-\frac{\sigma_{13} \sigma_{11}}{\sigma_{1}^{2}}-\frac{\sigma_{123}}{\sigma_{2}}+\frac{\sigma_{13} \sigma_{12}}{\sigma_{2} \sigma_{11}^{2}} \\
& +s \frac{\sigma_{133}}{\sigma_{1}}-s \frac{\sigma_{13}^{2}}{\sigma_{1}^{2}}+\frac{\sigma_{1} \sigma_{134}}{\sigma_{2}^{2}}+\frac{\sigma_{13} \sigma_{14}}{\sigma_{2}^{2}}
\end{aligned}
$$

in the same way as for $\varphi$ although, here, we also need to use the series expansion for $s$ as $t \rightarrow \infty$. The highest order derivative in this expression is $\sigma_{113}$ which has weight $7+7+2=16$ and so the Taylor series we obtain is valid to order 7 . We find

$$
\lim _{\mathbf{u} \rightarrow \mathbf{0}}\left[\frac{\mathrm{d}}{\mathrm{d} u_{1}} \Psi(\mathbf{u})\right]=\lim _{\xi \rightarrow 0}\left[-\lambda_{2}-2 \lambda_{1} \xi^{3}-3 \lambda_{0} \xi^{6}+O\left(\xi^{8}\right)\right]
$$

which is regular as $\mathbf{u}$ tends to $\mathbf{0}$. Thus the functions $\varphi_{2}$ and

$$
\frac{\mathrm{d}}{\mathrm{d} u_{1}}\left(-\frac{1}{3} \frac{1}{\lambda_{0}} \Psi(\mathbf{u})\right)
$$

have the same series expansion near the pole $\mathbf{u}=\mathbf{u}_{0}$ and are regular everywhere else on $\Theta_{1}$. It follows that we can write

$\left[\varphi_{2}\right] \mathrm{d} u_{1}+A_{2} \mathrm{~d} u_{1}+A_{3} \mathrm{~d} u_{2}+A_{4} \mathrm{~d} u_{4}=\left[\frac{\mathrm{d}}{\mathrm{d} u_{1}}\left(-\frac{1}{3} \frac{1}{\lambda_{0}} \Psi(\mathbf{u})\right)\right] \mathrm{d} u_{1}+\mathbf{B}^{\mathrm{T}} \mathrm{d} \mathbf{u}$

for some vector of constants $\mathbf{B}^{\mathrm{T}}=\left(B_{1}, B_{2}, B_{3}, B_{4}\right)$. 


\subsection{Evaluation of the vector $\mathbf{B}$}

We can now evaluate the vector $\mathbf{B}$ for the trigonal case using the same technique as for the higher genus hyperelliptic reductions.

Consider the Abelian differential

$$
\left[\varphi_{2}-\frac{\mathrm{d}}{\mathrm{d} u_{1}}\left(-\frac{1}{3} \frac{1}{\lambda_{0}} \Psi(\mathbf{u})\right)\right] \mathrm{d} u_{1}
$$

By definition $\mathrm{d} u_{1}$ is a first kind Abelian differential and so has zeros of degree $(2 g-2)=6$ and no poles on $\mathbf{T}_{4}$. From the calculations above we know that

$$
F=\left[\varphi_{2}-\frac{\mathrm{d}}{\mathrm{d} u_{1}}\left(-\frac{1}{3} \frac{1}{\lambda_{0}} \Psi(\mathbf{u})\right)\right]
$$

is regular on $\mathbf{T}_{4}$ and so $F$ must be a constant. If we compare the expansions of $\varphi_{2}$ and

$$
\frac{\mathrm{d}}{\mathrm{d} u_{1}}\left(-\frac{1}{3} \frac{1}{\lambda_{0}} \Psi(\mathbf{u})\right)
$$

near $\mathbf{u}=\mathbf{0}$, (83) and (89), we see that

$$
F=-\frac{1}{3} \frac{\lambda_{2}}{\lambda_{0}}
$$

and so identity (90) can be written

$$
-\frac{1}{3} \frac{\lambda_{2}}{\lambda_{0}} \mathrm{~d} u_{1}=\left[\left(B_{1}-A_{2}\right)+\left(B_{2}-A_{3}\right) t+B_{3} s+\left(B_{4}-A_{4}\right) t^{2}\right] \frac{\mathrm{d} t}{3 s^{2}} .
$$

To evaluate the vector $\mathbf{B}$ we look at the expansion of (91) as $t$ tends to infinity. The values of $\mathrm{d} u_{i}$ are given by equations (47) - (50). The left hand side is therefore

$$
\left(\frac{1}{3} \frac{\lambda_{2}}{\lambda_{0}}\right) \xi^{6}+O\left(\xi^{9}\right)
$$

and the right hand side becomes

$$
\begin{aligned}
& \left(A_{4}-B_{4}\right)+\left(-B_{3}\right) \xi+\left(\frac{2}{3} \lambda_{4}\left(B_{4}-A_{4}\right)+A_{3}-B_{2}\right) \xi^{3}+\left(\frac{1}{3} \lambda_{4} B_{3}\right) \xi^{4} \\
& +\left[\left(\frac{2}{3} \lambda_{3}-\frac{5}{9} \lambda_{4}^{2}\right)\left(B_{4}-A_{4}\right)+\frac{2}{3} \lambda_{4}\left(B_{2}-A_{3}\right)+A_{2}-B_{1}\right] \xi^{6}+O\left(\xi^{7}\right) .
\end{aligned}
$$

Matching coefficients of $\xi$, we find

$$
\begin{aligned}
& B_{4}=A_{4}, \\
& B_{3}=0, \\
& B_{2}=A_{3}, \\
& B_{1}=A_{2}-\frac{1}{3} \frac{\lambda_{2}}{\lambda_{0}}
\end{aligned}
$$

and so equation (90) becomes

$$
\begin{aligned}
& {\left[\varphi_{2}\right] \mathrm{d} u_{1}+A_{2} \mathrm{~d} u_{1}+A_{3} \mathrm{~d} u_{2}+A_{4} \mathrm{~d} u_{4}} \\
& =\left[\frac{\mathrm{d}}{\mathrm{d} u_{1}}\left(-\frac{1}{3} \frac{1}{\lambda_{0}} \frac{\sigma_{13}}{\sigma_{1}}\right)\right] \mathrm{d} u_{1}+\left(A_{2}-\frac{1}{3} \frac{\lambda_{2}}{\lambda_{0}}(\mathbf{u})\right) \mathrm{d} u_{1}+A_{3} \mathrm{~d} u_{2}+A_{4} \mathrm{~d} u_{4} .
\end{aligned}
$$




\subsection{Explicit formula for the trigonal reduction}

From the definition of $\lambda_{0}(26)$, we set

$$
K=3 \lambda_{0}^{2 / 3} \text {. }
$$

Substituting

$$
p=P_{6}-\frac{1}{t}=P_{6}+\frac{\sigma_{2}}{\sigma_{1}}(\mathbf{u}) \quad \mathbf{u} \in \Theta_{1}
$$

into (13), we have

$$
\begin{aligned}
\lambda(p)= & p+\int_{\infty}^{p}\left(\varphi\left(p^{\prime}\right)-1\right) \mathrm{d} p^{\prime} \\
= & \left(P_{6}+\frac{\sigma_{2}}{\sigma_{1}}(\mathbf{u})\right)+3 \lambda_{0}^{2 / 3} \int_{0}^{\frac{1}{P_{6}-p}}\left[\left(A_{2}-\frac{1}{3} \frac{\lambda_{2}}{\lambda_{0}}\right) \mathrm{d} u_{1}+A_{3} \mathrm{~d} u_{2}+A_{4} \mathrm{~d} u_{4}\right] \\
& +3 \lambda_{0}^{2 / 3} \int_{0}^{\frac{1}{P_{6}-p}}\left[\frac{\mathrm{d}}{\mathrm{d} u_{1}}\left(-\frac{1}{3} \frac{1}{\lambda_{0}} \frac{\sigma_{13}}{\sigma_{1}}(\mathbf{u})\right)\right] \mathrm{d} u_{1}-\int_{0}^{\frac{1}{P_{6}-p}} \frac{\mathrm{d} t}{t^{2}} \\
= & \left(P_{6}+\frac{\sigma_{2}}{\sigma_{1}}(\mathbf{u})\right)+ \\
& 3 \lambda_{0}^{2 / 3}\left[\left(A_{2}-\frac{1}{3} \frac{\lambda_{2}}{\lambda_{0}}\right) u_{1}+A_{3} u_{2}+A_{4} u_{4}-\frac{1}{3} \frac{1}{\lambda_{0}} \frac{\sigma_{13}}{\sigma_{1}}(\mathbf{u})\right] \\
& -\left[\frac{\sigma_{2}}{\sigma_{1}}(\mathbf{u})\right]+\widetilde{C}
\end{aligned}
$$

where the $A_{i}$ are defined in equation (25). To calculate the constant $\widetilde{C}$ we recall that the expansion of $\lambda(p)$ as $p$ tends to infinity is

$$
\lim _{p \rightarrow \infty} \lambda(p)=p+O\left(\frac{1}{p}\right)
$$

From the identity

$$
p=P_{6}+\frac{\sigma_{2}}{\sigma_{1}}(\mathbf{u}) \quad \mathbf{u} \in \Theta_{1}
$$

we see that $p \rightarrow \infty$ is equivalent to $\sigma_{1}(\mathbf{u}) \rightarrow 0$. The Taylor series for equation (93) is thus calculated in terms of $w_{i}=\left(\mathbf{u}-\mathbf{u}_{0}\right) \cdot \mathbf{e}_{i}$. We can then use the substitutions (63) - (65) to rewrite $w_{j}(j=2,3,4)$ in terms of $w_{i}$. We have

$\lim _{p \rightarrow \infty}[\lambda(p)-p]=$

$\lim _{\mathbf{u} \rightarrow \mathbf{u}_{0}}\left\{3 \lambda_{0}^{2 / 3}\left[\left(A_{2}-\frac{1}{3} \frac{\lambda_{2}}{\lambda_{0}}\right) u_{1}+A_{3} u_{2}+A_{4} u_{4}-\frac{1}{3} \frac{1}{\lambda_{0}} \frac{\sigma_{13}}{\sigma_{1}}(\mathbf{u})\right]-\frac{\sigma_{2}}{\sigma_{1}}(\mathbf{u})\right\}$.

Calculating the Taylor series for the first terms gives

$$
\begin{aligned}
\lim _{\mathbf{u} \rightarrow \mathbf{u}_{0}} & \left\{3 \lambda_{0}^{2 / 3}\left[\left(A_{2}-\frac{1}{3} \frac{\lambda_{2}}{\lambda_{0}}\right) u_{1}+A_{3} u_{2}+A_{4} u_{4}\right]-\frac{1}{\lambda_{0}^{1 / 3}} \frac{\sigma_{13}}{\sigma_{1}}(\mathbf{u})\right\} \\
= & {\left[-\frac{1}{3} \frac{1}{\lambda_{0}^{2 / 3}}\right] \frac{1}{w_{1}} } \\
& +3 \lambda_{0}^{2 / 3}\left[\left(A_{2}-\frac{1}{3} \frac{\lambda_{2}}{\lambda_{0}}\right) u_{0,1}+A_{3} u_{0,2}+A_{4} u_{0,4}+c_{0}\right]+O\left(w_{1}\right)
\end{aligned}
$$


where $c_{0}$ is

$$
\begin{aligned}
c_{0}= & \frac{1}{6\left(\lambda_{0} \sigma_{11}+\lambda_{0}^{4 / 3} \sigma_{13}\right)^{2}}\left[\left(6 A_{2} \lambda_{0}^{2} \sigma_{11}^{2}-2 \lambda_{0}^{5 / 3} \lambda_{2} \sigma_{13}^{2}+6 A_{2} \lambda_{0}^{8 / 3} \sigma_{13}^{2}-4 \lambda_{0}^{4 / 3} \lambda_{2} \sigma_{11} \sigma_{13}\right) u_{0,1}\right. \\
& +\left(6 A_{3} \lambda_{0}^{8 / 3} \sigma_{13}-2 \lambda_{2} \lambda_{0} \sigma_{11}^{2}+6 A_{3} \lambda_{0}^{2} \sigma_{11}^{2}\right) u_{0,2} \\
& +\left(6 A_{4} \lambda_{0}^{2} \sigma_{11}^{2}+6 A_{4} \lambda_{0}^{8 / 3} \sigma_{13}^{2}+12 A_{4} \lambda_{0}^{7 / 3} \sigma_{11} \sigma_{13}\right) u_{0,3}+\left(12 A_{4} \lambda_{0}^{7 / 3} \sigma_{11} \sigma_{13}\right) u_{0,4} \\
& +6 A_{4} \lambda_{0}^{2} \sigma_{11}^{2}-2 \lambda_{0} \sigma_{113} \sigma_{11}-2 \lambda_{0}^{4 / 3} \sigma_{113} \sigma_{11}+6 A_{4} \lambda_{0}^{8 / 3} \sigma_{13}^{2}+12 A_{2} \lambda_{0}^{7 / 3} \sigma_{13} \sigma_{11} \\
& \left.+3 \lambda_{0}^{5 / 3} \sigma_{12} \sigma_{13}+\lambda_{0} \sigma_{13} \sigma_{111}-\lambda_{0}^{5 / 3} \sigma_{13} \sigma_{133}+\lambda_{0} \lambda_{3} \sigma_{13}^{2}+12 A_{3} \lambda_{0}^{7 / 3} \sigma_{11} \sigma_{13}\right]
\end{aligned}
$$

Using substitutions (77) - (82) we find

$$
c_{0}=-\frac{3}{\lambda_{0}} \frac{\sigma_{23}}{\sigma_{2}}\left(\mathbf{u}_{0}\right)
$$

and so

$$
\begin{aligned}
& \lim _{\mathbf{u} \rightarrow \mathbf{u}_{0}}\left\{3 \lambda_{0}^{2 / 3}\left[\left(A_{2}-\frac{1}{3} \frac{\lambda_{2}}{\lambda_{0}}\right) u_{1}+A_{3} u_{2}+A_{4} u_{4}\right]-\frac{1}{\lambda_{0}^{1 / 3}} \frac{\sigma_{13}}{\sigma_{1}}(\mathbf{u})\right\} \\
& =\left[-\frac{1}{3} \frac{1}{\lambda_{0}^{2 / 3}}\right] \frac{1}{w_{1}} \\
& \quad+\left\{3 \lambda_{0}^{2 / 3}\left[\left(A_{2}-\frac{1}{3} \frac{\lambda_{2}}{\lambda_{0}}\right) u_{0,1}+A_{3} u_{0,2}+A_{4} u_{0,4}\right]-\frac{9}{\lambda_{0}^{1 / 3}} \frac{\sigma_{23}}{\sigma_{2}}\left(\mathbf{u}_{0}\right)\right\}+O\left(w_{1}\right) .
\end{aligned}
$$

The Taylor series for the second term in equation (94) is

$$
\begin{gathered}
\lim _{\mathbf{u} \rightarrow \mathbf{u}_{0}}\left[-\frac{\sigma_{2}}{\sigma_{1}}(\mathbf{u})\right]=\left[-\frac{\sigma_{2}}{\sigma_{11}+\lambda_{0}^{1 / 3} \sigma_{13}}\right] \frac{1}{w_{1}}+ \\
{\left[\frac { 1 } { 2 ( \sigma _ { 1 1 } + \lambda _ { 0 } ^ { 1 / 3 } \sigma _ { 1 3 } ) ^ { 2 } } \left(-2 \sigma_{11} \sigma_{12}-\lambda_{0}^{1 / 3} \sigma_{12} \sigma_{13}-2 \lambda_{0}^{1 / 3} \sigma_{11} \sigma_{33}-2 \lambda_{0}^{1 / 3} \sigma_{11} \sigma_{23}\right.\right.} \\
\left.\left.-2 \lambda_{0}^{2 / 3} \sigma_{13} \sigma_{23}+2 \lambda_{3} \sigma_{2} \sigma_{13}+\lambda_{0}^{2 / 3} \sigma_{2} \sigma_{133}+\sigma_{2} \sigma_{111}+2 \lambda_{0}^{1 / 3} \sigma_{2} \sigma_{113}+3 \lambda_{0}^{2 / 3} \sigma_{2} \sigma_{12}\right)\right]+O\left(w_{1}\right)
\end{gathered}
$$

again, using the substitutions (77) - (82) for the second and third order sigma derivatives, this becomes

$$
\lim _{\mathbf{u} \rightarrow \mathbf{u}_{0}}\left[-\frac{\sigma_{2}}{\sigma_{1}}(\mathbf{u})\right]=\left[\frac{1}{3} \frac{1}{\lambda_{0}^{2 / 3}}\right] \frac{1}{w_{1}}+\left[-\frac{1}{3} \frac{\lambda_{1}}{\lambda_{0}}\right]+O\left(w_{1}\right) .
$$

Since

$$
\left.\lim _{p \rightarrow \infty}[\lambda(p)-p)\right]=O\left(\frac{1}{p}\right)
$$

we set the constant $\widetilde{C}$ to be

$$
\widetilde{C}=-3 \lambda_{0}^{2 / 3}\left[\left(A_{2}-\frac{1}{3} \frac{\lambda_{2}}{\lambda_{0}}\right) u_{0,1}+A_{3} u_{0,2}+A_{4} u_{0,4}\right]+\frac{9}{\lambda_{0}^{1 / 3}} \frac{\sigma_{23}}{\sigma_{2}}\left(\mathbf{u}_{0}\right)+\frac{1}{3} \frac{\lambda_{1}}{\lambda_{0}} \cdot(98)
$$

To summarise, the mapping we require is given by the following result: 
Theorem 5.1 The Schwartz-Christoffel mapping

$$
\lambda(p)=p+\int_{\infty}^{p}\left(\frac{\prod_{i=1}^{4}\left(p-\hat{p}_{i}\right)}{\prod_{i=1}^{6}\left(p-P_{i}\right)}-1\right) \mathrm{d} p^{\prime},
$$

is given explicitly as follows.

Rather than the coordinates $(p, y)$ on the cyclic $(3,6)$ curve

$$
\Gamma=\left\{(p, y): y^{3}=\prod_{i=1}^{6}\left(p-P_{i}\right)\right\},
$$

we define new coordinates $(t, s)$ by:

$$
\begin{aligned}
& p=P_{6}-\frac{1}{t}, \\
& P_{i}=P_{6}-\frac{1}{T_{i}} \quad i=1 \ldots 5, \\
& s=y t^{2} K \\
& K^{3}=\prod_{i=1}^{5}\left(P_{6}-P_{i}\right) .
\end{aligned}
$$

Define $\lambda_{i}$ by the equation

$$
\sum_{i=1}^{6} \lambda_{i} t^{i}=-\frac{\prod_{i=1}^{6}\left[\left(P_{6}-P_{i}\right) t-1\right]}{\prod_{i=1}^{5}\left(P_{6}-P_{1}\right)} .
$$

and set $\mathbf{A}^{\mathbf{T}}=\left(A_{1}, A_{2}, A_{3}, A_{4}\right)$ where the $A_{i}$ are defined by

$$
\sum_{i=1}^{4} A_{i} t^{i}=\prod_{i=1}^{4}\left[\left(P_{6}-\hat{p}_{i}\right) t-1\right] .
$$

The image of $\Gamma, \mathbf{T}_{4}$ is then given by the cyclic $(3,5)$ curve

$$
\mathbf{T}_{4}=\left\{(t, s): s^{3}=t^{5}+\sum_{i=0}^{4} \lambda_{i} t^{i}\right\}
$$

We then define the restriction to $\mathbf{T}_{4}$ of the Abel map $\mathbf{u}$, with image $\Theta_{1} \subset \operatorname{Jac}\left(\mathbf{T}_{4}\right)$, by

$$
\begin{aligned}
& u_{1}=\int_{\infty}^{t} \frac{\mathrm{dt}^{\prime}}{3 s^{\prime 2}} \\
& u_{2}=\int_{\infty}^{t} \frac{t^{\prime} \mathrm{dt}^{\prime}}{3 s^{\prime 2}} \\
& u_{3}=\int_{\infty}^{t} \frac{s^{\prime} \mathrm{dt}^{\prime}}{3 s^{\prime 2}} \\
& u_{4}=\int_{\infty}^{t} \frac{t^{\prime 2} \mathrm{dt}^{\prime}}{s^{\prime 2}}
\end{aligned}
$$

The inversion of these mappings is given by:

$$
p=P_{6}+\frac{\sigma_{2}}{\sigma_{1}}(\mathbf{u}) .
$$


Then, with $\mathbf{u}=\left(u_{1}, u_{2}, u_{3}, u_{4}\right) \in \Theta_{1}$ and $\sigma_{1}\left(\mathbf{u}_{0}\right)=0$, we have:

$$
\begin{aligned}
\lambda(p)= & 3 \lambda_{0}^{2 / 3}\left(A_{2}-\frac{1}{3} \frac{\lambda_{2}}{\lambda_{0}}\right)\left(u_{1}-u_{0,1}\right)+A_{3}\left(u_{2}-u_{0,2}\right)+A_{4}\left(u_{4}-u_{0,4}\right) \\
& -\frac{1}{3} \frac{1}{\lambda_{0}^{1 / 3}} \frac{\sigma_{13}}{\sigma_{1}}(\mathbf{u})+\frac{9}{\lambda_{0}^{1 / 3}} \frac{\sigma_{23}}{\sigma_{2}}\left(\mathbf{u}_{0}\right)+\frac{1}{3} \frac{\lambda_{1}}{\lambda_{0}}
\end{aligned}
$$

on the sheet of the Riemann surface

$$
\left\{(p, y): y^{3}=\prod_{i=1}^{6}\left(p-P_{i}\right)\right\}
$$

associated with the relation $p \rightarrow+\infty \Leftrightarrow \mathbf{u} \rightarrow+\mathbf{u}_{0}$.

\section{Acknowledgements}

We are very grateful to Prof. V.Z. Enolski for many valuable discussions, for explaining some of his results to us, and for drawing our attention to many references, particularly Jorgenson's paper [23]. We wish to thank Prof. J.C. Eilbeck and Prof. Yoshihiro Onishi for their help, leading to the detection of an error in an earlier version of this work. I am particularly grateful to Prof. Eilbeck for his work verifying that the $C 20$, given below, is correct. 


\section{Appendix A. Expansion of $\sigma(\mathbf{u})$ near $\mathbf{u}=\mathbf{0}$}

For $\mathbf{u}$ near $\mathbf{u}=\mathbf{0}$, the expansion of sigma is an even function (see Onishi, [30], Lemma (5.4)):

$$
\begin{aligned}
\sigma(\mathbf{u})= & C_{8}\left(u_{1}, u_{2}, u_{3}, u_{4}\right)+C_{11}\left(u_{1}, u_{2}, u_{3}, u_{4}\right)+C_{14}\left(u_{1}, u_{2}, u_{3}, u_{4}\right)+C_{17}\left(u_{1}, u_{2}, u_{3}, u_{4}\right) \\
& +C_{20}\left(u_{1}, u_{2}, u_{3}, u_{4}\right)+O\left(u_{4}^{23}\right) .
\end{aligned}
$$

where

$$
\begin{aligned}
C_{8}= & u_{4}^{8}+448 u_{2}^{2}+448 u_{2} u_{3} u_{4}^{2}-56 u_{3}^{2} u_{4}^{4}-112 u_{3}^{4}-448 u_{1} u_{4}, \\
C_{11}= & \frac{1}{5} \lambda_{4}\left[-112 u_{3}^{5} u_{4}+16 u_{2} u_{4}^{7}+u_{4}^{9} u_{3}+2240 u_{2}^{2} u_{3} u_{4}-56 u_{4}^{5} u_{3}^{3}\right] \\
C_{14}= & -\frac{28}{5} \lambda_{3} u_{3}^{6} u_{4}^{2}+\left(\frac{13}{15} \lambda_{3}+\frac{8}{15} \lambda_{4}^{2}\right) u_{2} u_{3} u_{4}^{8}+\left(-\frac{1}{54600} \lambda_{4}^{2}+\frac{1}{21840} \lambda_{3}\right) u_{4}^{14} \\
& +\left(\frac{32}{15} \lambda_{3}-\frac{8}{15} \lambda_{4}^{2}\right) u_{1} u_{4}^{7}+\left(\frac{56}{15} \lambda_{4}^{2}+\frac{28}{15} \lambda_{3}\right) u_{2}^{2} u_{4}^{6}+\left(\frac{1}{60} \lambda_{3}+\frac{1}{75} \lambda_{4}^{2}\right) u_{3}^{2} u_{4}^{10} \\
& +336 \lambda_{3} u_{2}^{2} u_{3}^{2} u_{4}^{2}+448 \lambda_{3} u_{2}^{3} u_{3}-\frac{336}{5} \lambda_{3} u_{2} u_{3}^{5}+\left(-\frac{7}{15} \lambda_{3}-\frac{14}{15} \lambda_{4}^{2}\right) u_{3}^{4} u_{4}^{6} \\
& -56 \lambda_{3} u_{2} u_{3}^{3} u_{4}^{4},
\end{aligned}
$$

$$
\begin{aligned}
C_{17}= & \left(\frac{49}{85800} \lambda_{4} \lambda_{3}-\frac{3}{5720} \lambda_{2}-\frac{2}{10725} \lambda_{4}^{3}\right) u_{2} u_{4}^{13}+\left(\frac{56}{5} \lambda_{4} \lambda_{3}-\frac{56}{5} \lambda_{2}\right) u_{2}^{3} u_{4}^{5} \\
& +\left(\frac{1}{15} \lambda_{2}-\frac{2}{75} \lambda_{4}^{3}-\frac{2}{15} \lambda_{4} \lambda_{3}\right) u_{3}^{5} u_{4}^{7}+\left(\frac{1}{132} \lambda_{2}+\frac{1}{825} \lambda_{4}^{3}-\frac{1}{825} \lambda_{4} \lambda_{3}\right) u_{3}^{3} u_{4}^{11} \\
& +\left(\frac{1}{16016} \lambda_{2}+\frac{19}{3003000} \lambda_{4}^{3}-\frac{17}{600600} \lambda_{4} \lambda_{3}\right) u_{3} u_{4}^{15} \\
& +\left(-\frac{56}{5} \lambda_{4} \lambda_{3}-\frac{56}{5} \lambda_{2}\right) u_{2} u_{3}^{6} u_{4}+\left(\frac{4}{5} \lambda_{2}+\frac{8}{3} \lambda_{4} \lambda_{3}+\frac{8}{15} \lambda_{4}^{3}\right) u_{2}^{2} u_{3} u_{4}^{7} \\
& +\left(-\frac{98}{15} \lambda_{2}-\frac{42}{5} \lambda_{4} \lambda_{3}\right) u_{2} u_{3}^{4} u_{4}^{5}+\lambda_{2} u_{1} u_{3} u_{4}^{8}+\frac{56}{5} \lambda_{2} u_{1} u_{2} u_{4}^{6} \\
& +224 \lambda_{2} u_{1} u_{2} u_{3}^{2} u_{4}^{2}-\frac{56}{3} \lambda_{2} u_{1} u_{3}^{3} u_{4}^{4}-\frac{112}{5} \lambda_{2} u_{1} u_{3}^{5}-\frac{28}{15} \lambda_{2} u_{3}^{7} u_{4}^{3} \\
& +448 \lambda_{2} u_{1} u_{2}^{2} u_{3}-\frac{112}{3} \lambda_{2} u_{2}^{2} u_{3}^{3} u_{4}^{3}+\left(-\frac{1}{10} \lambda_{2}+\frac{3}{10} \lambda_{4} \lambda_{3}\right) u_{2} u_{3}^{2} u_{4}^{9} \\
& +\left(224 \lambda_{2}+224 \lambda_{4} \lambda_{3}\right) u_{2}^{3} u_{3}^{2} u_{4},
\end{aligned}
$$




$$
\begin{aligned}
& C_{20}=112 \lambda_{3}^{2} u_{2}^{3} u_{3}^{3} u_{4}^{2}+112 \lambda_{1} u_{1}^{2} u_{3}^{2} u_{4}^{2}+\frac{28}{5} \lambda_{1} u_{1}^{2} u_{4}^{6} \\
& +\left(\frac{1}{50} \lambda_{3}^{2}+\frac{2}{15} \lambda_{1}-\frac{4}{75} \lambda_{4} \lambda_{2}\right) u_{3}^{10}+\left(-\frac{79}{8763955200} \lambda_{3}^{2}+\frac{1}{29213184} \lambda_{1}\right. \\
& \left.-\frac{1}{73032960} \lambda_{4} \lambda_{2}-\frac{31}{13693680000} \lambda_{4}^{4}+\frac{31}{2738736000} \lambda_{4}^{2} \lambda_{3}\right) u_{4}^{20} \\
& +\left(-\lambda_{1}+\frac{1}{5} \lambda_{4} \lambda_{2}+\frac{2}{5} \lambda_{4}^{2} \lambda_{3}+\frac{13}{20} \lambda_{3}^{2}\right) u_{2}^{2} u_{3}^{2} u_{4}^{8} \\
& +\left(\frac{1}{528} \lambda_{1}+\frac{1}{1320} \lambda_{4} \lambda_{2}+\frac{1}{19800} \lambda_{4}^{4}-\frac{79}{158400} \lambda_{3}^{2}+\frac{1}{19800} \lambda_{4}^{2} \lambda_{3}\right) u_{3}^{4} u_{4}^{12} \\
& +\left(-\frac{211}{115315200} \lambda_{3}^{2}+\frac{23}{18018000} \lambda_{4}^{4}+\frac{1}{87360} \lambda_{4} \lambda_{2}\right. \\
& \left.+\frac{1}{384384} \lambda_{1}-\frac{173}{35035000} \lambda_{4}^{2} \lambda_{3}\right) u_{3}^{2} u_{4}^{16} \\
& +\left(-56 \lambda_{1}+14 \lambda_{3}^{2}\right) u_{2}^{4} u_{4}^{4}+\left(-\frac{1}{5} \lambda_{4} \lambda_{2}+\frac{3}{40} \lambda_{3}^{2}-\frac{13}{30} \lambda_{1}\right) u_{3}^{8} u_{4}^{4} \\
& +\left(\frac{112}{15} \lambda_{1}-\frac{112}{15} \lambda_{4} \lambda_{2}-\frac{84}{5} \lambda_{3}^{2}\right) u_{2}^{2} u_{3}^{6} \\
& +\left(168 \lambda_{3}^{2}+224 \lambda_{1}\right) u_{2}^{4} u_{3}^{2} \\
& +\left(-\frac{4}{32175} \lambda_{4}^{2} \lambda_{3}+\frac{1}{64350} \lambda_{4}^{4}+\frac{8}{32175} \lambda_{3}^{2}-\frac{1}{17160} \lambda_{4} \lambda_{2}-\frac{1}{8580} \lambda_{1}\right) u_{1} u_{4}^{13} \\
& +\left(-\frac{1}{150} \lambda_{4}^{2} \lambda_{3}+\frac{1}{60} \lambda_{1}-\frac{13}{1200} \lambda_{3}^{2}+\frac{1}{150} \lambda_{4} \lambda_{2}\right) u_{3}^{6} u_{4}^{8} \\
& +\left(-\frac{1}{4950} \lambda_{4}^{4}-\frac{1}{4950} \lambda_{4}^{2} \lambda_{3}-\frac{1}{132} \lambda_{1}+\frac{79}{39600} \lambda_{3}^{2}+\frac{1}{3300} \lambda_{4} \lambda_{2}\right) u_{2}^{2} u_{4}^{12} \\
& +\left(-\frac{56}{15} \lambda_{4} \lambda_{2}-\frac{112}{15} \lambda_{1}\right) u_{1} u_{3}^{6} u_{4} \\
& +\left(\frac{28}{15} \lambda_{3}^{2}+\frac{56}{15} \lambda_{4}^{2} \lambda_{3}\right) u_{4}^{6} u_{3} u_{2}^{3}+\left(-\frac{28}{5} \lambda_{1}-\frac{14}{5} \lambda_{4} \lambda_{2}\right) u_{1} u_{3}^{4} u_{4}^{5} \\
& +\left(\frac{1}{5} \lambda_{1}+\frac{1}{10} \lambda_{4} \lambda_{3}\right) u_{1} u_{3}^{2} u_{4}^{9}+\left(-\frac{14}{25} \lambda_{4}^{2} \lambda_{3}-\frac{7}{25} \lambda_{3}^{2}-\frac{28}{25} \lambda_{4} \lambda_{2}\right) u_{2} u_{3}^{5} u_{4}^{6} \\
& +\left(\frac{56}{5} \lambda_{4} \lambda_{2}+\frac{112}{5} \lambda_{1}\right) u_{1} u_{2}^{2} u_{4}^{5}+\left(-\frac{28}{3} \lambda_{4} \lambda_{2}-28 \lambda_{1}-21 \lambda_{3}^{2}\right) u_{2}^{2} u_{3}^{4} u_{4}^{4} \\
& +\left(\frac{5}{72072} \lambda_{4}^{2} \lambda_{3}+\frac{1}{300300} \lambda_{4} \lambda_{2}+\frac{2}{3003} \lambda_{1}\right. \\
& \left.-\frac{401}{3603600} \lambda_{3}^{2}-\frac{1}{64350} \lambda_{4}^{4}\right) u_{2} u_{3} u_{4}^{14} \\
& +\left(\frac{1}{75} \lambda_{4}^{2} \lambda_{3}+\frac{1}{75} \lambda_{4} \lambda_{2}+\frac{1}{60} \lambda_{3}^{2}\right) u_{2} u_{3}^{3} u_{4}^{10}+\left(224 \lambda_{4} \lambda_{2}+448 \lambda_{1}\right) u_{1} u_{2}^{2} u_{3}^{2} u_{4}
\end{aligned}
$$




$$
\begin{aligned}
& +\left(\frac{16}{5} \lambda_{4} \lambda_{2}+\frac{32}{5} \lambda_{1}\right) u_{1} u_{2} u_{3} u_{4}^{7}+\left(-\frac{16}{15} \lambda_{4} \lambda_{2}-\frac{12}{5} \lambda_{3}^{2}-\frac{128}{15} \lambda_{1}\right) u_{2} u_{3}^{7} u_{4}^{2} \\
& +448 \lambda_{1} u_{1}^{2} u_{2} u_{3}
\end{aligned}
$$

\section{References}

[1] D J Benney Some properties of long nonlinear waves Stud. Appl. Math 5245 (1973).

[2] V E Zakharov Funct. Anal. Appl. 1489 (1980);

V E Zakharov On the Benney equations Physica D 3193 (1981).

[3] J Gibbons Collisionless Boltzmann equations and integrable moment equations Physica D 3503 (1981).

[4] B A Kupershmidt and Yu I Manin Long wave equation with a free surface I: Conservation laws and solutions Funct. Anal. Appl. 1131 (1997)

[5] J Gibbons and S P Tsarev Reductions of the Benney equations Phys. Lett. A 21119 (1996).

[6] J Gibbons and S P Tsarev Conformal maps and reductions of the Benney equations Phys. Lett. A 258 (1999). 263

[7] $\mathrm{L} \mathrm{Yu} \mathrm{and} \mathrm{J} \mathrm{Gibbons} \mathrm{The} \mathrm{initial} \mathrm{value} \mathrm{problem} \mathrm{for} \mathrm{reductions} \mathrm{of} \mathrm{Lax} \mathrm{equations} \mathrm{Inverse} \mathrm{Problems}$ 16605 (2000).

[8] S Baldwin and J Gibbons Hyperelliptic reduction of the Benney moment equations J. Phys. A: Math. Gen. 368393 ( 2003).

[9] S Baldwin and J Gibbons Higher genus hyperelliptic reductions of the Benney equations, J. Phys. A: Math. Gen. 37 5341-5354 (2004).

[10] Crowdy, D., The Benney hierarchy and the Dirichlet boundary problem in two dimensions. Phys. Lett. A 343 (2005), no. 4, 319-329.

[11] Crowdy, D., Genus-N algebraic reductions of the Benney hierarchy within a Schottky model, J. Phys. A: Math. Gen., 38, No 50 (16 December 2005), 10917-10934.

[12] Wiegmann, P.B., Zabrodin, A., Conformal maps and integrable hierarchies, Comm. Math. Phys. 213, 523-538, (2000).

[13] Kostov, I.K., Krichever, I.M., Mineev-Weinstein, M., Wiegmann, P.B., Zabrodin, A. The $\tau$ function for analytic curves, in: Random matrix models and their applications, 285-299, Math. Sci. Res. Inst. Publ. 40, CUP, Cambridge, (2001).

[14] Gibbons J and Kodama Y Solving Dispersionless Lax Equations, in: Singular Limits of Dispersive Waves (New York: Plenum) pp 61-6 (1994).

[15] Eilbeck, J. C., Enolskii, V. Z., Leykin, D. V., On the Kleinian construction of abelian functions of canonical algebraic curves. SIDE III-symmetries and integrability of difference equations (Sabaudia, 1998), 121-138, CRM Proc. Lecture Notes, 25, Amer. Math. Soc., Providence, RI, (2000).

[16] V.M. Buchstaber, V.Z. Enolskii and D.V. Leykin, Uniformisation of Jacobi varieties of trigonal curves and nonlinear differential equations, Func. Anal. App., 34159 (2000) .

[17] Y. Onishi Determinant expressions in Abelian functions for purely trigonal curves of degree four. Unpublished preprint: math.NT/0503696.

[18] K Matsumoto Theta constants associated with the cyclic triple coverings of the complex projective line branching at six points, Publ. Res. Inst. Math. Sci. 37, no. 3, 419-440, (2001).

[19] V M Buchstaber, V Z Enolskii and D V Leykin, Kleinian functions, hyperelliptic Jacobians and applications, Reviews in Mathematics and Mathematical Physics 10:2 1 (1997).

[20] M S Alber and Yu. N. Fedorov, Wave solutions of evolution equations and Hamiltonian flows on nonlinear subvarieties of generalised Jacobians, J. Phys. A 33 8409-8425 (2000).

[21] Enolskii, V. Z., Pronine, M., Richter, P. H., Double pendulum and $\theta$-divisor, J. Nonlinear Sci. 13 no. 2, 157-174 (2003). 
[22] E D Belokolos, A I Bobenko, V Z Enolskii, A R Its and V B Matveev (1994) Algebro-Geometrical Approach to Nonlinear Integrable Equations (Berlin: Springer)

[23] J Jorgenson On the directional derivatives of the theta function along its divisor. Israel J. of Math. 77, 273-284 (1992).

[24] Fay, J. D., Theta functions on Riemann surfaces, Lecture notes in mathematics, 352, (1973).

[25] Grant, D., A generalisation of a formula of Eisenstein, Proc. Lond. Math. Soc., 62, 121-132, (1991).

[26] K Weierstrass and HA Schwartz, Formeln und Lehrsätze zum Gebrauche der elliptischen Functionen, (Springer, Berlin, 1893).

[27] M Abramowitz and IA Stegun, Handbook of Mathematical Functions, 18.5.6, p.635, (Dover, New York, 1965).

[28] HF Baker, Multiply Periodic Functions, (CUP, Cambridge, 1907).

[29] Buchstaber, V. M., Leykin, D. V. Lie algebras associated with $\sigma$-functions, and versal deformations. (Russian) Uspekhi Mat. Nauk 57 (2002), no. 3(345), 145-146; translation in Russian Math. Surveys 57 (2002), no. 3, 584-586.

[30] Onishi, Y., Determinantal expressions in Abelian functions for purely Pentagonal curves of degree six, preprint, (2006). 